\title{
Research Progress on Artificial Protective Films for Lithium Metal Anodes
}

\author{
Dongdong Liu, Chao Chen, Xunhui Xiong * \\ School of Environment and Energy, South China University of Technology, Guangzhou 510006, China.
}

\begin{abstract}
In the early 1990s, Sony launched the first commercial lithium ion battery (LIB), which achieved great success in energy storage systems. The current commercially used insertion anode, graphite, is approaching its capacity limit ( 372 $\left.\mathrm{mAh} \cdot \mathrm{g}^{-1}\right)$, and is inadequate to satisfy the ever-increasing energy demand for power grids and large-scale energy storage systems. In order to address this challenge, lithium metal anodes have been the focus of considerable research effort in recent years, and are regarded as the most promising anode materials because of their extremely high theoretical capacity $\left(3860 \mathrm{mAh} \cdot \mathrm{g}^{-1}\right)$, lowest electrode potential $(-3.04 \mathrm{~V}$ vs. standard hydrogen electrode), and low density $\left(0.534 \mathrm{~g} \cdot \mathrm{cm}^{-3}\right)$. For example, the theoretical energy densities of lithium-sulfur batteries and lithium-air batteries are as high as 2567

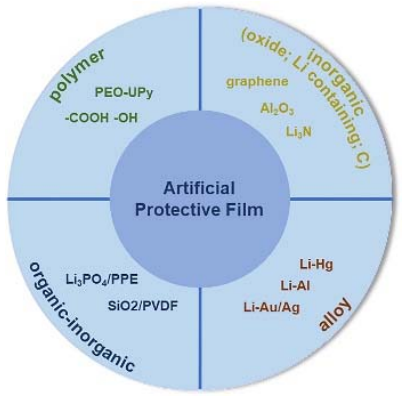
and $3505 \mathrm{Wh} \cdot \mathrm{kg}^{-1}$, respectively. However, the uncontrollable dendrite growth during cycling leads to low coulombic efficiency and puncture of the separator, causing a short circuit or even explosion of the battery, thereby seriously hindering the development of the lithium metal anode. Many solutions have been proposed to inhibit dendrite growth, including the use of electrolyte additives, solid electrolytes, and artificial protective films. During charging and discharging, the solid electrolyte interphase (SEI) plays an important role in lithium metal anodes. However, the infinite volume changes of the electrode during plating/stripping processes result in breakage of the SEI film, which continuously consumes the electrolyte and lithium metal. Designing an artificial interface on the surface of lithium metal anodes has been considered as a simple and efficient strategy to control lithium deposition behavior, and is achieved by precoating a protective layer on the surface of lithium metal. An ideal artificial protective film should possess high ionic conductivity, chemical stability, and excellent mechanical strength, in order to prevent side reactions between lithium metal and the electrolyte and realize dendrite-free lithium metal anodes with a long cycle life and high coulombic efficiencies. In this paper, the research progress on artificial protective films for lithium metal anodes in recent years is reviewed. Further, the structural characteristics and preparation methods of various protective films are introduced in detail, including polymer protective films, inorganic protective films, organic-inorganic composite protective films, and alloy protective films. The mechanisms of various protective films toward the suppression of dendrite growth are summarized. Existing challenges and future research directions are also proposed, which together provide a reference for promoting the use of lithium metal in high-energy batteries.
\end{abstract}

Key Words: Lithium metal; Anode; Dendrite; Artificial protective film; High energy density

\footnotetext{
Received: August 25, 2020; Revised: September 21, 2020; Accepted: September 21, 2020; Published online: October $12,2020$.

*Corresponding author. Email: esxxiong@scut.edu.cn. Tel.: +86-20-39381203.

The project was supported by the National Natural Science Foundation of China (51874142,), the Fundamental Research Funds for the Central Universities (2019JQ09), and the Tip-top Scientific and Technical Innovative Youth Talents of Guangdong Special Support Program (2019TQ05L903), and the Young Elite Scientists Sponsorship Program by CAST (2019QNRC001).

国家自然科学基金(51874142)，中央高校基本科研基金(2019JQ09)，广东特支计划 “科技创新青年拔尖人才”项目(2019TQ05L903)和中国科协 青年托举人才项目 (2019QNRC001)资助
}

(C) Editorial office of Acta Physico-Chimica Sinica 


\section{锂金属负极人造保护膜的研究进展}

刘冬冬, 陈超, 熊训辉 ${ }^{*}$

华南理工大学环境与能源学院, 广州 510006

摘要: 金属锂因其具有极高的理论容量 $\left(3860 \mathrm{mAh} \cdot \mathrm{g}^{-1}\right)$ 、最低的电极电位 $(-3.04 \mathrm{~V} v \mathrm{~s}$. 标准氢电极)和低的密度 $(0.534$ $\mathrm{g} \cdot \mathrm{cm}^{-3}$ ), 被认为是最具潜力的负极材料。但循环过程中不可控的枝晶生长及不稳定的固体电解质相界面膜所引起的安全 隐患和电池库伦效率低等问题严重阻碍了锂金属负极的发展。通过在电极表面构建人造保护膜可以有效调控锂离子沉积 行为, 因此人造保护膜的构建是一种简单高效抑制锂枝晶生长的策略。本综述将从聚合物保护膜、无机保护膜、有机-无 机复合保护膜和合金保护膜总结了人造保护膜的构建方法、抑制锂枝晶生长机理, 为促进高比能锂金属电池的商业化应 用提供借鉴参考作用。

关键词: 金属锂; 负极; 枝晶; 人造保护膜; 高比能 中图分类号: 0646

\section{1 引言}

首款商用锂离子电池(lithium-ion batteries, LIBs)于上世纪 90 年代初面世, 与镍镉电池相比 较, 锂离子电池在提供相同电量的情况下, 具有体 积更小, 质量更轻的优势 1 , 在能源行业取得了巨 大的成功。目前, 商业用的锂离子电池主要以石墨 为负极, 理论容量为 $372 \mathrm{mAh} \cdot \mathrm{g}^{-1}$, 其实际能量密 度也随着电池行业的发展逐渐趋近于理论极限 值 ${ }^{2,3}$ 。然而, 随着电子产品和电动汽车的快速发展 和普及, 传统石墨负极在比容量和能量密度方面 已经不能满足日益增长的社会需求 ${ }^{4-6}$, 因此发展 更高效的锂电池成为研究热点。

从铅酸电池到镍镉电池、镍氢电池和锂离子 电池, 这些商用电池能量密度的增加速度过于缓 慢。能量密度是指单位质量或单位体积所能提供 的电能总量 7 。金属理不仅具有极高的理论比容量 (3860 $\left.\mathrm{mAh} \cdot \mathrm{g}^{-1}\right)$ 和低电极电位 $(-3.04 \mathrm{~V} v s$. 标准氢电 极), 还是自然界中密度最小的金属 $\left(0.534 \mathrm{~g} \cdot \mathrm{cm}^{-3}\right)^{8,9}$ 。 这些优势促进了金属锂用于高能量密度电池的研 究设计, 即锂金属电池(lithium metal batteries, LMBs)。其中, 以锂硫电池和锂空电池为代表的 LMBs, 其理论能量密度分别高达 2567 和 3505 $\mathrm{Wh} \cdot \mathrm{kg}^{-1} 10,11$, 因此金属锂被认为是下一代最具有 竞争力的负极材料。

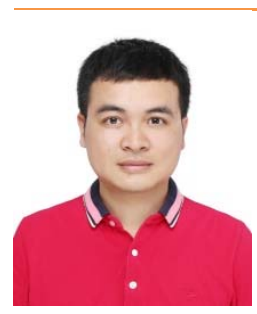

熊训辉, 教授, 博士生导师。主要从事 有色金属能源材料的可控制备及储能 机理研究。

\section{2 锂金属负极存在的主要问题及措施}

尽管锂金属负极对实现高能量密度电池的应 用展现出巨大潜力, 但由于诸多问题尚不能实现 实际应用。如图1a, 其存在的问题主要包括: (1)枝 晶的形成。由于不均匀的锂离子传导及尖端效应, 造成锂的不均匀沉积, 形成锂枝晶。当枝晶生长到 一定程度时会刺穿隔膜, 造成电池短路、起火甚至 爆炸等安全隐患。如果枝晶断裂形成 “死锂”, 则 会导致容量损失 ${ }^{12,13}$ 。(2)相较于石墨和硅这类插层 电极, 金属锂是一种无宿主电极材料, 在电镀/剥 离的过程中会产生无限的体积变化, 而尚未解决 的枝晶问题进一步加剧了体积效应的严重程度, 导致不稳定的固态电解质界面(solid electrolyte interphases, SEI)容易发生破裂，极活泼的金属锂 与电解液反应, 反复形成新的 SEI膜, 消耗电解液和 金属锂, 最终造成库伦效率低、界面阻抗增大 ${ }^{14-17}$ 。 综上可见, 枝晶问题是阻碍锂金属高比能电池面 世的巨大挑战。然而, 因枝晶的生长机理涉及到动 力学、热力学和表面化学等领域, 影响因素十分复 杂, 直至目前仍然无法用某种单一的模型解释枝 晶生长行为。目前, 锂枝晶的形成原因和生长过程 有以下三种模型:

(1)空间电荷模型：1990年, Chazalviel提出的 空间电荷理论是目前接受最为广泛的枝晶成核理 论 18,19 。在沉积过程中, 随着负极附近 $\mathrm{Li}^{+}$的逐渐消 耗, 而引起局部浓差极化, 使得电极和电解质界面 产生 “空间电荷”, 这种空间电荷产生的空间电场 会诱发理枝晶的生长。当 $\mathrm{Li}^{+}$浓度为零时, 枝晶开 始生长, 式(1)中, $\tau$ 为枝晶生长的初始时间, $D$ 为 双极扩散系数, $e$ 为电荷, $C_{0}$ 为电解液初始浓度, $J$ 为有效电流密度, $\mu_{\mathrm{a}}$ 和 $\mu_{\mathrm{Li}}{ }^{+}$分别为阴离子和 $\mathrm{Li}^{+}$迁 

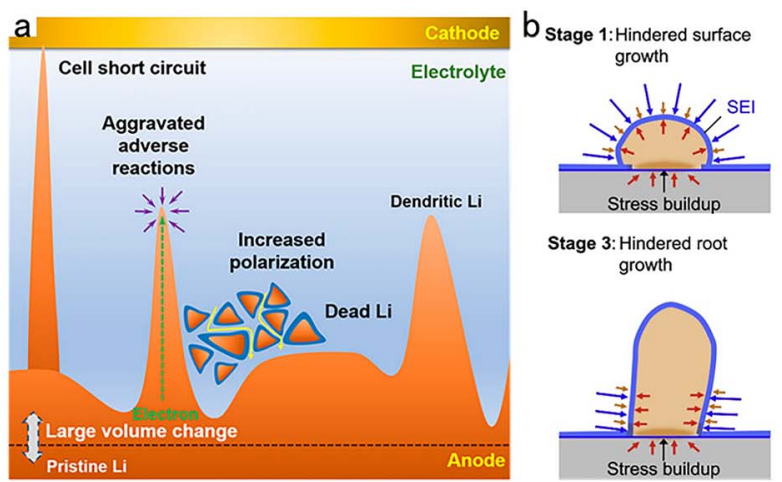
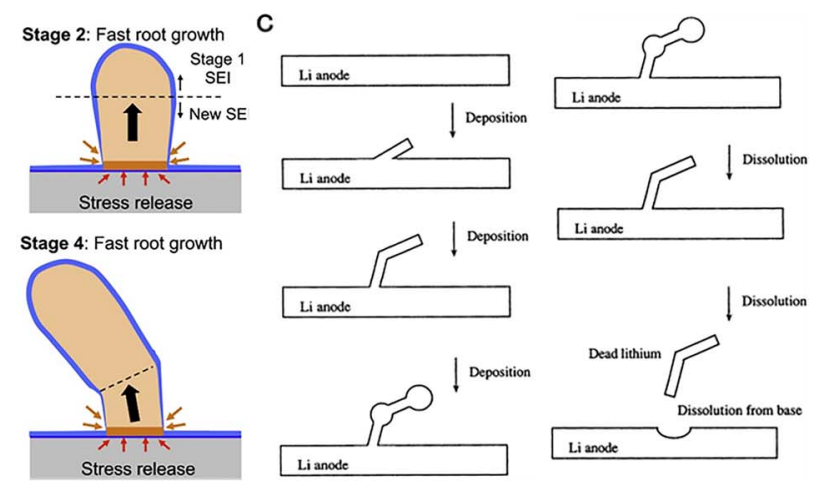

图 1 (a) 锂金属负极存在的问题示意图 4; (b)锂枝晶根系生长机理的示意图(adapted from. copyright: Elsevier) ${ }^{23}$; (c)锂沉积溶解过程示意图 26

Fig. 1 (a) Scheme of dilemma for Li metal anode in rechargeable batteries ${ }^{4}$; (b) schematic illustration explaining root growth mechanism of lithium whiskers (adapted from. copyright: Elsevier) ${ }^{23}$; (c) schematic diagram of lithium deposition and dissolution ${ }^{26}$.

移率。 $\tau$ 值越小时, 代表枝晶越容易形成。可见, 电流密度越高且 $\mathrm{Li}^{+}$扩散速度越小时, 枝晶越快出 现。因此。可以通过3D负极降低电极表面的电流 密度或者提高 $\mathrm{Li}^{+}$迁移率来延缓枝晶的生长时间。

$$
\tau=\pi D \frac{e C_{0}\left(\mu_{\mathrm{a}}+\mu_{\mathrm{Li}^{+}}\right)^{2}}{2 J \mu_{\mathrm{a}}^{2}}
$$

(2) SEI诱导模型: 在电池首次充放电中, 活泼 的金属锂与电解液反应形成SEI膜, 其表现出离子 传导性和电子绝缘性, 因此锂沉积发生在 SEI膜 下。电池循环过程中, SEI膜因无法承受无限的体 积膨胀而容易发生破裂, 其裂纹处成为电化学热 点, 离子传输能力增强, 诱导丝状和树枝状锂生 长 ${ }^{20-22}$ 。除此之外, SEI膜的自身性质对枝晶的形 成也有影响。如图 $1 \mathrm{~b}$, 根据根生长机制, 锂枝晶的 生长可以分成 4 个阶段。第一, $\mathrm{Li}^{+}$要穿过 $\mathrm{SEI}$ 膜在 金属锂表面沉积, 因此扩散速度受SEI膜的限制, 其 将在SEI膜较薄的部位优先沉积, 在锂表面形成球 形锂核, 锂核的直径与生长时间的平方根成正比。 第二, 枝晶从根部开始生长, 最初形成的球状核被 推离根部。在此阶段, 枝晶的长度迅速增长, 但是 直径基本保持不变。第三, 由于SEI膜重新生成或 者厚度增加, 枝晶生长速度显著下降。第四, 枝晶 上形成扭结, 将枝晶分为 $\mathrm{L} 1$ 和 L2两段, 新生成的 L2 段继续从根部生长, $\mathrm{L}$ 1段基本保持不变。可见， $\mathrm{Li}^{+}$通过SEI膜的短程固相扩散过程对锂沉积起重 要作用。当电流密度较小时, $\mathrm{Li}^{+}$在SEI膜的扩散速 度相对较快, 锂沉积时直径和长度同步增长, 有利 于形成致密的表面; 当电流密度较大时, $\mathrm{Li}^{+}$在 $\mathrm{SEI}$ 膜的扩散速度非常慢, 锂倾向于在长度上增长, 宽 度上几乎不变，从而导致枝晶生长 ${ }^{23}$ 。

(3)沉积-溶解模型: 该模型阐述了锂的沉积溶
解过程(图1 $\mathrm{c}$ ), 由于金属锂具有较低的表面能和较 高的表面扩散势垒, 在锂沉积过程中, $\mathrm{Li}^{+}$在锂负 极表面扩散速度慢 ${ }^{2}$ 。在外接电源的供应下，电解 液中的 $\mathrm{Li}^{+}$通过SEI膜传输到锂金属表面, 其倾向于 在晶界处或者 SEI膜具有较高离子传导率的位点 集中沉积。由于沉积不均匀, 锂负极产生机械应 力, 造成SEI膜破裂, 金属锂穿过裂纹处并以晶须 的形式继续生长。经过长时间的沉积后, 锂负极被 晶须覆盖, 阻碍了 $\mathrm{Li}^{+}$的传输, 锂开始沉积在晶须 的尖端和扭结点, 诱导枝晶生长。该模型认为机械 应力是引起枝晶的主要原因, 并应用式(2)拉普拉 斯方程, 其中 $\Delta P$ 是表面压差, $\gamma$ 代表表面张力, $R_{1}$ 和 $R_{2}$ 正交曲率半径。基于这个模型, Yamaki等 25,26 提出当保护膜的表面张力大于 $0.2 \mathrm{~N} \cdot \mathrm{m}^{-1}$ 时, 可以 有效抑制枝晶。

$$
\Delta P=\gamma\left(\frac{1}{R_{1}}+\frac{1}{R_{2}}\right)
$$

针对以上问题, 国内外研究者们已经进行了 大量的研究实验, 主要包括电解液改性 ${ }^{27-29}$ 、固态 电解质 ${ }^{30-32}$ 、人造 SEI膜 ${ }^{33-37}$ 及 $3 \mathrm{D}$ 锂金属负极 ${ }^{38-41}$ 等。其中, 电解液添加剂相对于有机电解质来说, 应具有更高的还原电位，以确保金属锂优先与电 解液添加剂反应。因此, 在电池循环过程中电解液 添加剂可以认为是一种消耗品, 不利于电池长期 循环 ${ }^{42,43}$ 。不仅如此, 有机系液体电解质的易燃性 使其易与金属锂发生副反应，存在较大的安全隐 患。而固态电解质可以有效解决由有机系液体电 解质带来的问题。无论是聚合物固态电解质还是 无机陶瓷固态电解质，其具有的高剪切模量能够 机械抑制枝晶生长, 提高电池的安全性能。但是, 与有机系电解液相比, 固态电解质的离子传导率 
在室温下只有 $10^{-8}-10^{-5} \mathrm{~S} \cdot \mathrm{cm}^{-14}$ 。锂合金负极代 替锂金属可以有效控制枝晶问题, 然而由于循环 过程中体积变化太大, 合金负极的循环寿命受到 限制。为了解决电池的体积效应, 研究者们提出了 具有亚微米结构和高比表面积的3D锂金属负极, 金 属锂可以储存在3D结构中, 从而控制无限的体积 变化。同时, 其具有的高比表面积能显著降低电极 表面的电流密度, 抑制枝晶生长 ${ }^{44,45}$ 。但是 $3 \mathrm{D}$ 锂金 属负极无法有效避免金属锂与电解液所产生的非 法拉第反应。由于SEI膜的脆性和不均匀性直接影 响了锂沉积行为, 人造保护膜的研究越来越广泛。 人造保护膜是通过在电极表面涂覆某种或多种材 料以达到调控金属锂沉积行为的策略, 有效避免 了SEI膜在化学组成和空间分布等方面的不均匀 性 ${ }^{46-48}$ 。其具有良好的电化学稳定性和离子传导 性、优异的阻隔性能和柔韧性, 从而促进 $\mathrm{Li}^{+}$的均 匀传输, 缓解充放电循环过程中的体积变化并抑 制锂枝晶生长, 实现长循环寿命、高库伦效率的锂 金属负极。

\section{3 人造保护膜}

人造保护膜应用于锂金属负极已经引起了极 大的关注, 原位SEI膜是指向电解液中添加合适的 锂盐或溶剂促使锂金属负极在初始活化周期构建 更加稳定的SEI膜。然而, 在电池循环过程中电解 液添加剂逐渐被消耗, 对后续生成的SEI膜的组分 和厚度有所影响, 且通过优化电解液原位形成的 SEI膜机械稳定性差且不具备高模量, 难以机械抑 制枝晶生长 ${ }^{42,43}$ 。而通过化学气相沉积 (chemical vapor deposition, CVD) ${ }^{49}$ 、原子层沉积 (atomic layer deposition, ALD) ${ }^{50}$ 和磁控溅射 (magnetron sputtering) ${ }^{51}$ 等方法构建的非原位人造保护膜在厚 度和机械强度等方面可以实现高度可控。理想的 人造保护膜应满足以下要求: (1)高锂离子传导性、 促进 $\mathrm{Li}^{+}$均匀传输; (2) 良好的化学稳定性和柔韧 性, 适应电池循环期间的体积波动; (3)高弹性模 量, 以达到机械抑制枝晶生长的目的; (4)优异的 阻隔性能及电子绝缘性, 以阻止金属锂与电解液 之间的副反应。本文将根据锂金属表面保护膜的 成分, 从聚合物保护膜 ${ }^{52,53}$ 、无机保护膜 $54-56$ 、复 合保护膜 ${ }^{57-59}$ 和合金保护膜 60 四种非原位人造保 护膜进行概述。

\section{1 聚合物保护膜}

近几年, 聚合物保护膜因其制造过程方便且 具有优异的柔韧性和良好的稳定性, 能够承受电 池循环过程中无限的体积变化, 抑制枝晶生长, 从
而实现高倍率、长循环寿命的锂金属负极。不均匀 的锂离子传导和电荷在电极表面不均匀分布是导 致锂枝晶生长的主要原因。由于 $\mathrm{Li}^{+}$和极性基团 ( $-\mathrm{COOR} 、-\mathrm{OH} 、-\mathrm{F}$ 等)之间的强相互作用, 将 带有极性基团的聚合物层作为人造SEI膜能够有 效控制锂离子通量在锂金属负极表面均匀分布 ${ }^{61}$ 。 $\mathrm{Li}$ 等 ${ }^{62}$ 制备了聚乙烯醇(PVA)保护膜, PVA碳链骨 架上具有丰富的极性基团 $(-\mathrm{OH})$, 其与锂原子具 有高结合能(图2a-d)。在锂沉积过程中, 一旦过多 的 $\mathrm{Li}^{+}$在突起处还原为锂原子, 它们将从突起处脱 离, 并与邻近的 $-\mathrm{OH}$ 结合, 由此减轻尖端效应, 促进均匀的锂沉积。此外, 这类保护膜具有优异的 阻隔性能, 能够保护金属锂在空气中稳定存在一 段时间而不受腐蚀。Sun等 63 通过分子层沉积法 (MLD)制备了一种新型且超薄的聚艮有机膜作为 锂金属负极的保护层。聚嫝中含氮的极性基团可 以有效地调节锂离子通量, 促使锂均匀沉积。文中 使用碳酸盐电解质进行组装电池, 当电流密度增 加为 $3 \mathrm{~mA} \cdot \mathrm{cm}^{-2}$ 时, 由于枝晶快速生长刺破隔膜, 未保护的锂电极在循环 $62 \mathrm{~h}$ 后失效, 而聚腿膜保护 的锂电极能够稳定循环 $200 \mathrm{~h}$, 是未保护的锂金属 电极循环寿命的3倍, 在较高的电流密度下表现出 了优异的循环稳定性和循环寿命(图 $2 \mathrm{e}-\mathrm{h}$ )。根据 Chazalviel的空间电荷理论, 枝晶的生长速度 $v$ 可用 式(3)表示, 其中 $\mu_{\mathrm{a}}$ 表示阴离子的迁移率, $E_{0}$ 表示电 场 ${ }^{18}$ 。由此可见, 可以通过降低阴离子的迁移率来 减缓由空间电荷效应引起的枝晶生长。 $\mathrm{Li}^{\text {等 }}{ }^{64}$ 以丝 胶蛋白层作为人造 SEI膜, 其丰富的官能团 $\left(-\mathrm{COOH} 、-\mathrm{OH} 、-\mathrm{CO}-\mathrm{NH} 、-\mathrm{NH}_{2}\right)$ 不仅具有 亲锂性还能亲阴离子, 在调节锂离子通量的同时 缓解负极表面的阴离子浓度梯度, 在这些极性基 团的协同作用下, 电池在电流密度 $10 \mathrm{~mA} \cdot \mathrm{cm}^{-2}$ 及 沉积量为 $5 \mathrm{mAh} \cdot \mathrm{cm}^{-2}$ 的条件下稳定循环 160 个周 期。在锂硫电池中也展现出优异的循环性能和倍 率性能。

$$
v=-\mu_{\mathrm{a}} E_{0}
$$

大量的实验数据表明聚合物保护膜在锂金属 负极方面的应用已经取得了一定的成效。但这些 聚合物的离子传导率较差, 也不具备原位生成SEI 膜的自我修复特性, 导致聚合物保护膜的有效周 期限制了电池的循环寿命。如图 3 , 我们课题组 65 通过简单的滴涂法在锂金属负极表面构建了自稳 定、强粘附力的超分子聚合物保护膜, 该聚合物包 含脲基嘧啶酮(UPy)基团和聚环氧乙烷(PEO)侧链 段, 当与金属锂接触时, UPy将与锂自发反应, 原 位形成自稳定、致密的LiPEO-UPy保护膜, 起到增 

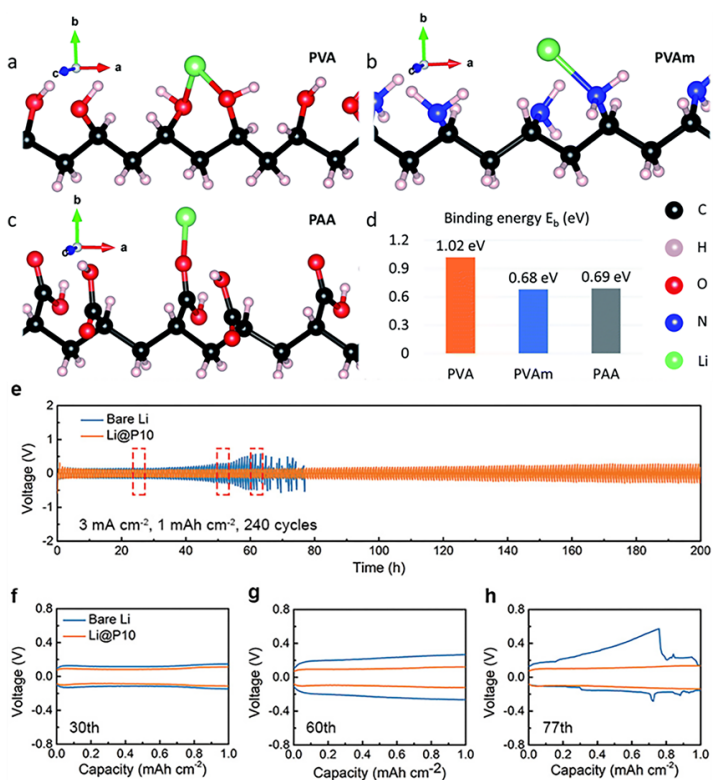

图 2 经几何优化的聚合物结构及其与 Li 原子的成键状态示意图(a) PVA，(b) PVAm，(c) PAA 和(d)三者之间的 结合能对比图 ${ }^{62}$; (e) $3 \mathrm{~mA} \cdot \mathrm{cm}^{-2} / 1 \mathrm{mAh} \cdot \mathrm{cm}^{-2}$ 下的电压分布曲线和 $3 \mathrm{~mA} \cdot \mathrm{cm}^{-2}$ 下详细的电压分布：(f) 30 个周期处,

(g) 60 个周期处和(h) 77 和周期处 63

Fig. 2 Schematic diagrams of the polymer structure and its bonding state with Li atoms after geometry optimization for (a) PVA, (b) PVAm, (c) PAA and (d) comparison of the corresponding binding energies $\left(E_{b}\right){ }^{62}$; (e) voltage-time curves at a higher current density of $3 \mathrm{~mA} \cdot \mathrm{cm}^{-2}$ for the capacity of $1 \mathrm{mAh} \cdot \mathrm{cm}^{-2}$ and Detailed voltage profiles at $3 \mathrm{~mA} \cdot \mathrm{cm}^{-2}$ for:

(f) 30th, (g) 60th, and (h) 77 th cycles ${ }^{63}$.

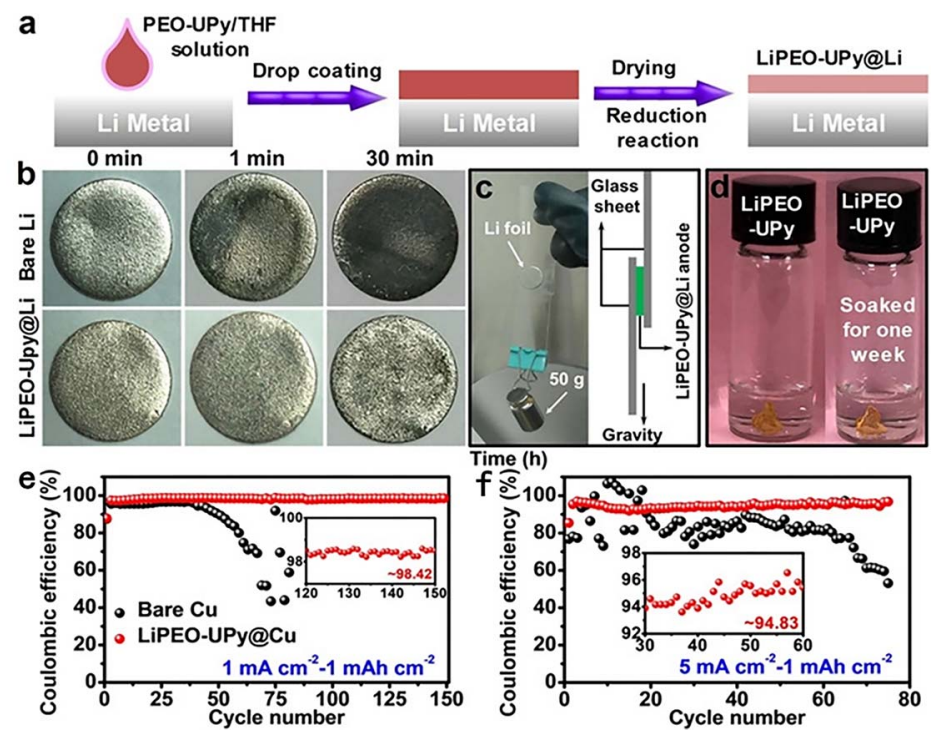

图 3 (a)锂金属表面 PEO-UPy 涂覆过程示意图; (b)未保护的锂电极和 LiPEO-UPy@Li 电极暴露在空气中不同时间的 照片; (c) PEO-UPy@Li 电极对 $50 \mathrm{~g}$ 质量的附着力试验; (d) LiPEO-UP 聚合物在 DOL/DME 溶液中保持完整; 铜电极和 LiPEO-UPy@Cu 电极在面容量为 $1 \mathrm{mAh} \cdot \mathrm{cm}^{-2}$ 下的库伦效率图(e) $1 \mathrm{~mA} \cdot \mathrm{cm}^{-2}$ 和(f) $5 \mathrm{~mA} \cdot \mathrm{cm}^{-2} 65$

Fig. 3 (a) Schematic diagram of PEO-UPy coating on Li metal surface; (b)photographs of bare Li and LiPEO-UPy@Li anodes exposed to ambient air for different durations; (c) adhesion test of PEO-UPy@Li anode against $50 \mathrm{~g}$ mass;

(d) LiPEO-UPy polymer kept integrity in DOL/DME solution; CEs of bare Cu and LiPEO-UPy@Cu electrodes with a fixed capacity of $1 \mathrm{mAh} \cdot \mathrm{cm}^{-2}$ at a current density of (d) $1 \mathrm{~mA} \cdot \mathrm{cm}^{-2}$ and (e) $5 \mathrm{~mA} \cdot \mathrm{cm}^{-2} 65$.

强电极/电解液界面稳定性、适应界面巨大波动的 作用。同时, $\mathrm{PEO}$ 与 $\mathrm{Li}^{+}$之间的静电作用可以延缓
$\mathrm{Li}^{+}$沉积并使之在电极表面均匀沉积。综合以上优 势, 电池在锂金属负极高利用率时仍具有 $95 \%$ 的库 
伦效率。针对聚合物膜如何进行改性设计以及聚 合物膜对电池的影响机制的问题。Cui等66研究了 几种具有不同化学性质和机械性能的聚合物保护 膜(图4a, b), 为聚合物膜对锂离子沉积过程的影 响提供了几点新的见解: 1) 保护膜厚度对锂沉积 过程具有显著影响, 保护膜过薄会影响锂成核过 程, 而过厚会限制 $\mathrm{Li}^{+}$的传输, 从而降低锂沉积的 均匀性。2)自愈合聚合物 (SHP) 和自愈合弹性体 (SHE)具有相似的化学性质, 但SHP是可流动的粘 弹性材料, SHE是共价交联的弹性固体。通过比较 SHP和SHE两种聚合物膜, 研究发现聚合物膜的力 学性能会影响锂离子的均匀沉积, 但其化学功能 性才是影响 $\mathrm{Li}$ 局部形态的主要因素。3)如图 4c所 示, 聚合物的介电常数和表面能共同影响着沉积 颗粒的尺寸, 交换电流随着聚合物膜介电常数的 增大而增大, 而低表面能的聚合物会产生更高的 界面能, 从而共同增大锂离子沉积时的颗粒尺寸。

\section{2 无机保护膜}

锂是一种极活泼的金属, 为稳定电解液/锂金 属界面, 研究者们开发了各类保护膜。然而保护膜 若不具有良好的机械性能, 在电池长久运行后枝 晶生长依然是最大的问题。相较于其他保护膜, 无 机保护膜展现出高机械强度和优异的离子传导 性, 研究表明, 保护膜的杨氏模量高于 $6 \mathrm{GPa}$ 时足 以通过机械阻力抑制枝晶生长 67,68 。因此, 无机保 护膜对于抑制枝晶具有重要意义。

\subsection{1 氧化物}

在锂沉积过程中, $\mathrm{Li}^{+}$更倾向于在低过电势或 低势垒的区域成核, 通过降低成核势垒以此减小 枝晶形成的驱动力 69,70 。因此构建亲锂性的保护层 能够降低锂成核过电位, 抑制枝晶生长 ${ }^{71}$ 。部分金 属氧化物都具有亲锂性, 且与电解液中的极性基团 具有很强的相互作用, 因而具有良好的润湿性 ${ }^{72-74}$ 。 $\mathrm{Al}_{2} \mathrm{O}_{3}$ 经常用于构建保护层应用在锂金属负极。 Noked 等 75 使用等离子体增强的原子层沉积法 (ALD)在金属锂表面制备了 $\mathrm{Al}_{2} \mathrm{O}_{3}$ 层, 但是就锂沉 积在 $\mathrm{Al}_{2} \mathrm{O}_{3}$ 层上还是层下的问题文中没有指明。但 是在Dasgupta等 ${ }^{76}$ 的工作中, 其证实了 $\mathrm{Li}^{+}$能够扩散 穿过 $\mathrm{Al}_{2} \mathrm{O}_{3}$ 层, 在层下发生沉积, 抑制枝晶生长。 但是这两项工作均未测量 $\mathrm{Al}_{2} \mathrm{O}_{3}$ 层厚度。Elam等 50 使用 $\mathrm{ALD}$ 技术, 以三甲基铝(TMA)和 $\mathrm{H}_{2} \mathrm{O}$ 为气相前 驱体交替脉冲在锂金属表面制备了 $\mathrm{Al}_{2} \mathrm{O}_{3}$ 薄膜。其 厚度与 ALD循环次数相关, 通过 X射线荧光光谱 (X-ray Fluorescence Spectrum, XRF) 测量 $\mathrm{Al}_{2} \mathrm{O}_{3}$ 薄 膜厚度, 最终采用 $\mathrm{ALD}$ 循环 20 次的 $\mathrm{Al}_{2} \mathrm{O}_{3}$ 层(厚度约 $4 \mathrm{~nm}$ )组装电池进行电化学测试。在 $1 \mathrm{~mA} \cdot \mathrm{cm}^{-2} / 1$ $\mathrm{mAh} \cdot \mathrm{cm}^{-2}$ 条件下循环 50 个周期后, 未保护的锂电 极表面变得高度多孔并伴随枝晶的生长。相反, $\mathrm{Al}_{2} \mathrm{O}_{3}$ 层修饰的锂电极表面平滑致密, 没有枝晶生 长的迹象(图5), 这是因为 $\mathrm{Al}_{2} \mathrm{O}_{3}$ 层增强了锂表面对 碳酸盐和醚类电解质的润湿性, 使电解液更加均 匀的覆盖在电极表面, 促进形成均匀稳定的 SEI

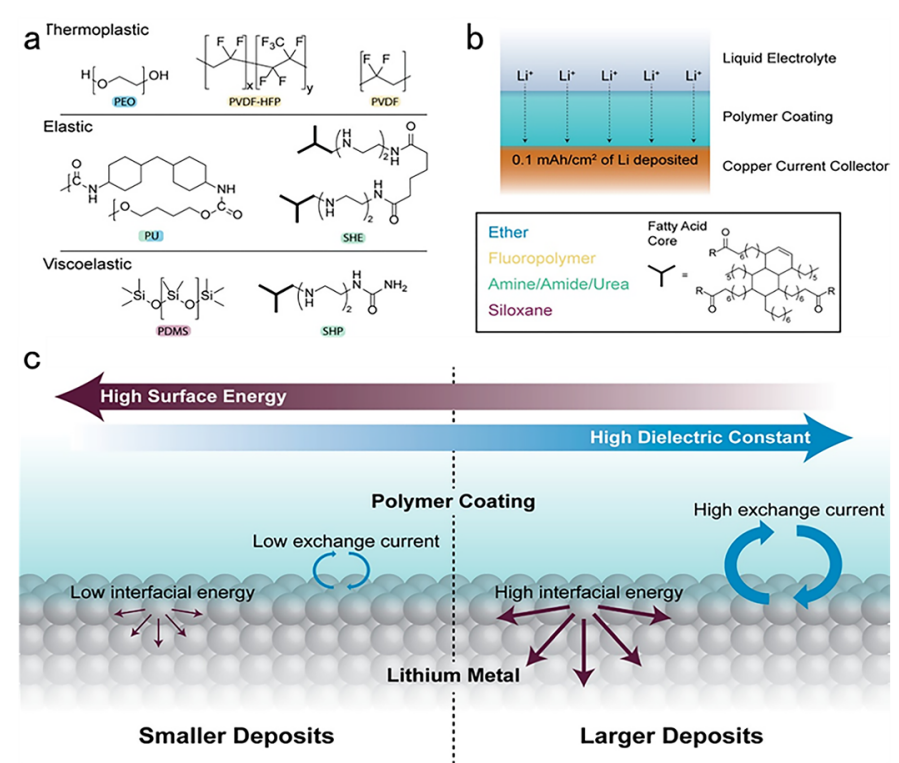

图 4 (a)几种聚合物保护膜的化学结构(标签的颜色与聚合物的化学官能团相对应); (b)用于研究聚合物保护膜下 锂金属初始生长阶段的条件; (c)聚合物保护膜影响锂金属沉积的因素原理图 66

Fig. 4 (a) Chemical structures of the polymer coatings used in this study, coloring of the label corresponds to the chemical functionality of the polymer; (b) diagram of the conditions used to study the initial stages of Li metal growth under polymer coatings; (c) schematic of the factors influencing Li metal deposition through a polymer coating ${ }^{66}$. 
(a)

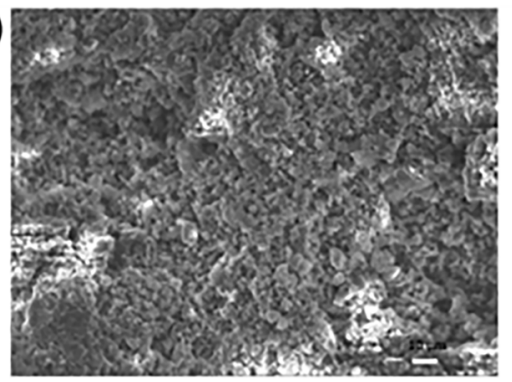

(c)

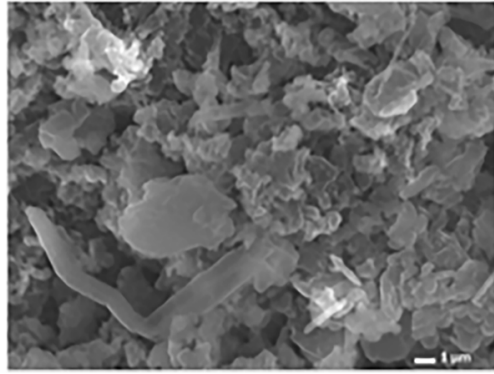

(b)

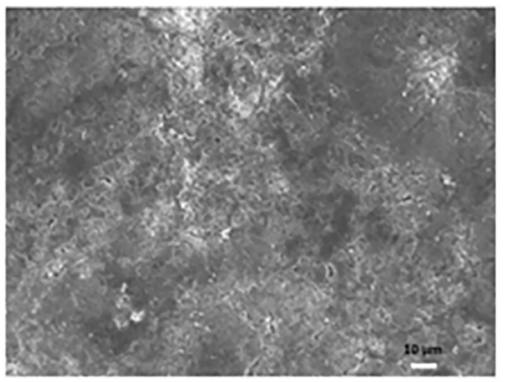

(d)

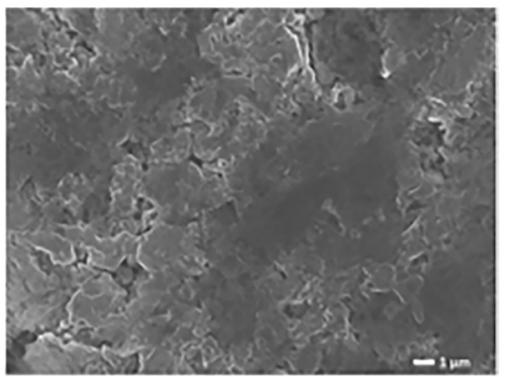

图 5 在 $1 \mathrm{~mA} \cdot \mathrm{cm}^{-2} / 1 \mathrm{mAh} \cdot \mathrm{cm}^{-2}$ 条件下对称电池经过 50 次充放电循环后锂表面的扫描电镜图像: 600 倍放大时

(a)未保护的锂电极; (b) $\mathrm{Al}_{2} \mathrm{O}_{3}$ 保护的锂电极和 5000 倍放大时(c)未保护的锂电极; (d) $\mathrm{Al}_{2} \mathrm{O}_{3}$ 保护的锂电极 50

Fig. 5 SEM images of Li surfaces following 50 charge-discharge cycles in symmetric cells using current rate of

$1 \mathrm{~mA} \cdot \mathrm{cm}^{-2}$ and capacity of $1 \mathrm{mAh} \cdot \mathrm{cm}^{-2}$ at 600 magnification (a) bare $\mathrm{Li}$ and (b) $\mathrm{Al}_{2} \mathrm{O}_{3} @ \mathrm{Li}$; and at 5000 magnification (c) bare $\mathrm{Li}$ and (d) $\mathrm{Al}_{2} \mathrm{O}_{3} @ \mathrm{Li}^{50}$.

膜。Dunn等77将锂箔浸没于四乙氧基硅烷(TEOS) 中, 在电极表面制备了多孔的 $\mathrm{SiO}_{2}$ 膜。未经TEOS 处理的锂电极表面在锂剥离后出现明显的凹坑, 在锂沉积后出现苔藓状区域; 而经TEOS处理的锂 电极在沉积过程中能够填补保护膜下方的空隙, 从而保持电极表面平滑完整。但是, 氧化物保护膜 的 $\mathrm{Li}^{+}$传导性较差, 且在电池充放电过程中较易发 生破裂, 与聚合物构造复合保护膜效果更佳。

\section{2 .2 含锂化合物}

含锂化合物被广泛应用于锂金属负极保护 层, 其中最为典型的是亲锂性的 $\mathrm{Li}_{3} \mathrm{~N} 。 \mathrm{Li}_{3} \mathrm{~N}$ 具有高 离子传导率(室温下为 $10^{-3}-10^{-4} \mathrm{~S} \cdot \mathrm{cm}^{-1}$ ), 有利于 界面 $\mathrm{Li}^{+}$均匀扩散, 抑制 $\mathrm{Li}^{+}$集中沉积。Wen等 ${ }^{78}$ 将 $\mathrm{Li}$ 与 $\mathrm{N}_{2}$ 气体在室温下直接化学反应, 在锂箔表面原 位形成 $\mathrm{Li}_{3} \mathrm{~N}$ 保护层。 $\mathrm{Li}_{3} \mathrm{~N}$ 不仅具有高的离子电导 率以促进 $\mathrm{Li}^{+}$的迁移, 而且能够形成稳定的 SEI膜以 阻止金属锂与电解液之间的副反应。在锂硫电池 中, $\mathrm{Li}_{3} \mathrm{~N}$ 层还能防止锂金属和多硫化物接触, 抑制 他们之间的腐蚀反应, 提高电池的循环寿命和安 全性(图6a, b)。在电流密度为 $0.2 C$ 且电解液中未添 加 $\mathrm{LiNO}_{3}$ 的条件下循环 200 个周期后, 受 $\mathrm{Li}_{3} \mathrm{~N}$ 保护 的电池平均库伦效率为 $91.4 \%$ 、容量保持率为 $79.7 \%$; 未受 $\mathrm{Li}_{3} \mathrm{~N}$ 保护的电池平均库伦效率为 $80.7 \%$ 、容量保持率只有 $37.2 \%$ 。卤化锂的扩散势 垒很低, 有利于 $\mathrm{Li}^{+}$的传输, 其中研究最为广泛的
是 $\mathrm{LiF}, \mathrm{LiF}$ 是 SEI膜中至关重要的成分, 具有强机 械性能和极佳的化学稳定性等优点。如图6c, Cui 等 ${ }^{79}$ 以含氟聚合物 $\mathrm{CYTOP}$ 为前驱体, 开发了一种 简便的表面氟化反应。CYTOP在较低的温度下分 解产生纯氟气体，与金属锂反应形成均匀致密的 $\mathrm{LiF}$ 层, 实现长循环后无枝晶锂负极。Zhao 等 ${ }^{80}$ 通 过金属锂与碘蒸汽直接反应, 在锂金属负极上生 成致密的LiI层, 提高锂负极界面稳定性。 $\mathrm{Li}_{3} \mathrm{PO}_{4}$ 保 护膜能够增强 $\mathrm{Li}^{+}$的传输, 其优异的化学稳定性确 保在电池循环过程中不易发生破裂, 保持结构 完整, 抑制电解质和金属锂之间的不利反应。Guo 等 ${ }^{81}$ 采用多聚磷酸(PPA)对金属锂进行预处理, 两 者发生原位反应制备了稳定均匀的 $\mathrm{Li}_{3} \mathrm{PO}_{4}$ 保护 膜, 其较高的杨氏模量(10-11 GPa)有效抑制枝晶 生长, 在锂/磷酸铁锂 $\left(\mathrm{LiFePO}_{4}\right)$ 电池中缓解电解液 对金属锂的腐蚀, 提高电化学性能(图6d, e)。 $\mathrm{PCl}_{3}$ 与金属锂在室温下反应生成 $\mathrm{Li}_{3} \mathrm{P}$ 和 $\mathrm{LiCl}, \mathrm{Yang}$ 等 82 利用这一反应在锂金属负极表面制备了 $\mathrm{Li}_{3} \mathrm{P} / \mathrm{LiCl}$ 涂层。 $\mathrm{Li}_{3} \mathrm{P}$ 作为锂离子良导体降低了界面阻抗, 与 次生相 $\mathrm{LiCl}$ 共同抑制锂硫电池的穿梭效应, 大大 提高电池稳定性。Yu等 ${ }^{83}$ 在低温下通过简单的气固 反应 $\left(\mathrm{SeS}_{2} / \mathrm{Li}\right.$ ) 制备了 $\mathrm{Li}_{2} \mathrm{~S} / \mathrm{Li}_{2} \mathrm{Se}(\mathrm{LSSe}$ ) 保护层(图 $7 \mathrm{a})$ 。 $\mathrm{Li}_{2} \mathrm{~S}$ 因具有高离子传导率已被应用于锂金属 负极, $\mathrm{Li}_{2} \mathrm{Se}$ 与 $\mathrm{Li}_{2} \mathrm{~S}$ 具有相似的化学性质, 且密度泛 函理论(Density Functional Theory, DFT)计算表明, 
a

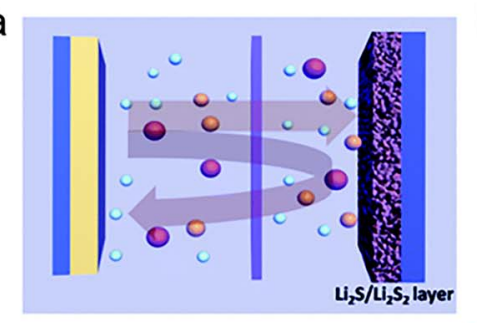

L Lithium ions $\rightarrow$ Polysulfides ions

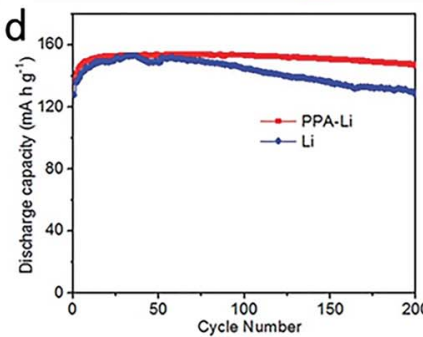

b

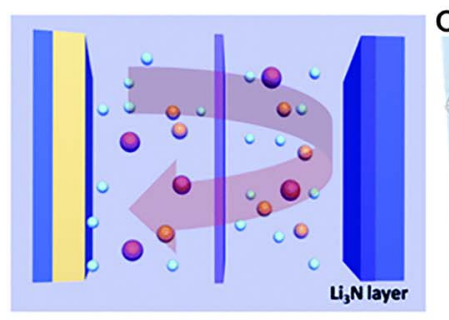

Sulfur cathode $\square$ Lithium anode
C

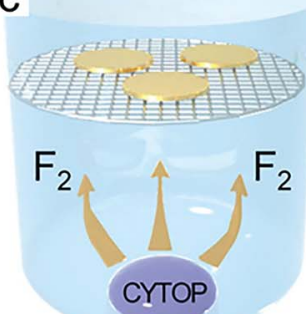

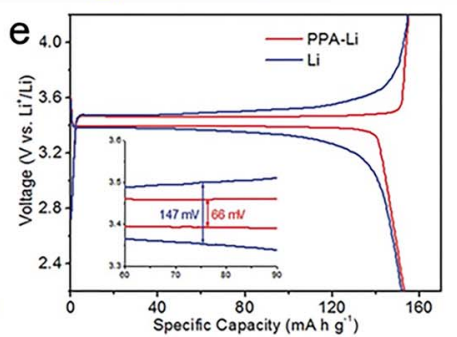

图 6 锂硫电池中锂金属负极的设计示意图(a)没有 $\mathrm{Li} i \mathrm{~N}$ 保护膜和(b)有 $\mathrm{Li} 3 \mathrm{~N}$ 保护膜 ${ }^{78}$; (c) $\mathrm{LiF}$ 涂层的制备示意图 ${ }^{79}$; (d)未保护的锂电极和 $\mathrm{Li}_{3} \mathrm{PO}_{4}$ 保护的锂电极在 $\mathrm{Li}_{\mid} \mid \mathrm{LiFePO}_{4}$ 电池中的循环性能图和(e) $0.5 \mathrm{C}$ 的充放电曲线, 插图是放大的部分曲线 81

Fig. 6 Schematic of the design of a lithium metal electrode in lithium-sulfur battery configurations, (a) a battery without the $\mathrm{Li}_{3} \mathrm{~N}$ layer and (b) a battery with the $\mathrm{Li}_{3} \mathrm{~N}$ layer ${ }^{78}$; (c) schematic diagram of preparation of LiF coating ${ }^{79}$; (d) cycling performance of the $\mathrm{Li} \mid \mathrm{LiFePO}_{4}$ battery system using $\mathrm{Li}$ metal and PPA-Li anodes and (e) the typical charge/discharge profiles after activation at a current rate of $0.5 C$, the inset of (e) shows enlarged profiles ${ }^{81}$.

a
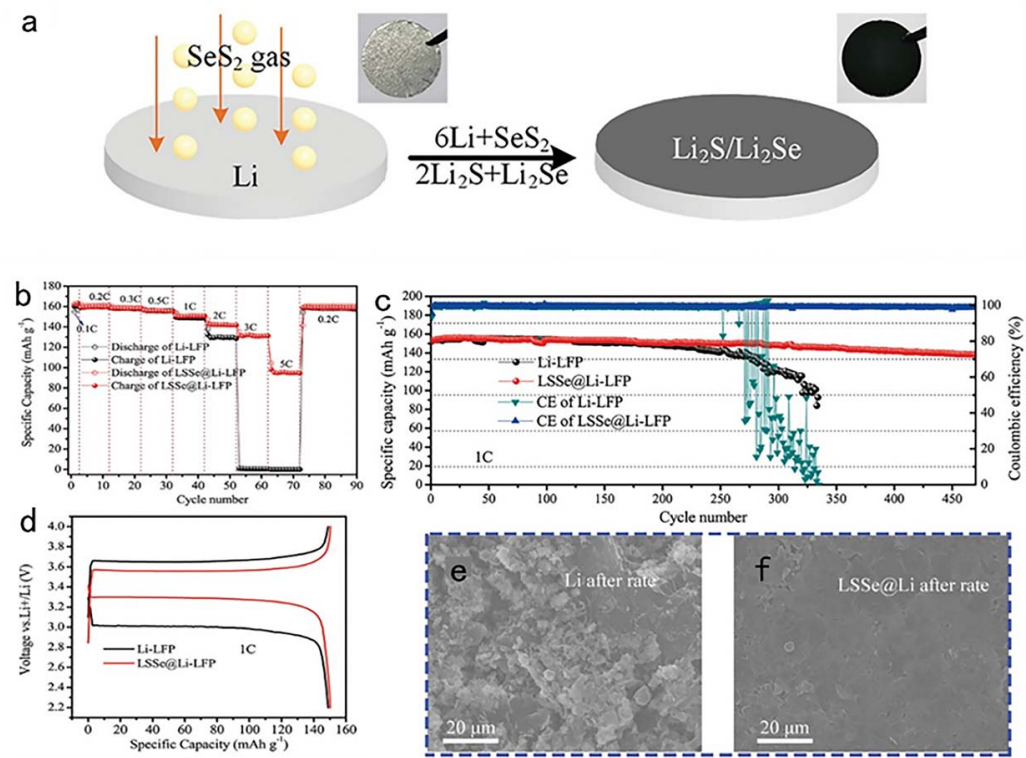

图 7 (a) LSSe@Li 负极制作工艺示意图; Li-LFP 和 LSSe@Li-LFP 电池的电化学性能图(b)倍率性能图; (c)倍率为 $1 C$ 时 的循环性能; (d)倍率为 $1 C$ 时的充放电曲线; 循环后扫描电镜图(e)未保护的锂电极和(f) LSSe@Li 电极 ${ }^{83}$

Fig. 7 (a) Schematic illustration of fabrication process of LSSe@Li anode; the electrochemical performance of Li-LFP and

LSSe@Li-LFP: (b) the rate performance, (c) the cycling performance at the rate of $1 C$, (d) the charge/discharge curves at the rate of $1 C$; the surface morphology of (e) bare Li and (f) LSSe@Li anode after rate cycling ${ }^{83}$.

$\mathrm{Li}_{2} \mathrm{Se}$ 在不同晶面的锂离子迁移势垒低于 $\mathrm{Li}_{2} \mathrm{~S}$, 说 明 $\mathrm{Li}_{2} \mathrm{Se}$ 的引入有利于锂离子迁移。在与 $\mathrm{LiFePO}_{4}$ 组 成的电池中具有更好的电池性能和形貌(图 7b-f)。 锂磷氧氮化物 (LiPON) 的弹性模量高达 $77 \mathrm{GPa}$ 且
具有优异的化学和电化学稳定性 ${ }^{84}$, 对构建稳定的 电极/电解液界面具有巨大潜力。 $\mathrm{Fu}$ 等 ${ }^{85}$ 采用氮等 离子体辅助电子束蒸发在锂金属表面沉积 LiPON 膜, 厚度约为 $250 \mathrm{~nm}$ 。该方法不需要使用大型靶材 
且沉积速率高 $\left(>66 \mathrm{~nm} \cdot \mathrm{min}^{-1}\right)$, 适合大规模生产。 LiPON包覆的锂金属负极在对称电池和锂硫电池 中均展现出优异的电化学性能。综上可见, 锂化合 物类保护膜具有促进 $\mathrm{Li}^{+}$的传输、刚性强度较大的 优点, 但是这类保护膜的柔韧性还需进一步提高, 以此适应电极的体积变化, 避免保护膜发生破裂。

\subsection{3 碳材料}

碳材料也已被开发用于锂金属负极保护, 它 有结晶和非晶态两种形式, 其中非晶态碳由 $s p^{2}$ 、 $s p^{3}$ 杂化碳组成, 具有高硬度、高弹性模量、化学 稳定等优异的性质, 能够有效抑制枝晶的生长, 且 在充放电循环过程中不易产生裂纹。

$\mathrm{Tu}$ 等 ${ }^{86}$ 利用磁控溅射在金属锂上制备非晶碳 膜, 厚度从 50-110 nm 可控, 保护膜越厚其抑制枝 晶的效果越好, 但同时也增加了 $\mathrm{Li}^{+}$传输的阻力, 因此通过控制磁控溅射的时间制备厚度适中的保 护膜至关重要。Cui等 ${ }^{87}$ 在锂金属负极上沉积了一 种高灵活性、互连的空心非晶碳纳米球层。该保护 膜具有优异的化学稳定性, 能与金属锂稳定相存。 其杨氏模量高达200 GPa足以抑制枝晶生长且不 会增加 $\mathrm{Li}^{+}$传输的阻力。碳层与集流体之间存在一 定的结合力, 在循环过程中碳层能够上下移动适 应锂沉积的体积变化。在电流密度 $1 \mathrm{~mA} \cdot \mathrm{cm}^{-2}$ 及沉 积量 $1 \mathrm{mAh} \cdot \mathrm{cm}^{-2}$ 的条件下循环 150 个周期后库伦 效率高达 $99 \%$ 。如图 $8 \mathrm{a}, \mathrm{Xie}^{\text {等 }}{ }^{88}$ 在室温下用碱金属 直接还原氧化石墨烯, 以均匀的氧化石墨烯为涂
料, 利用喷涂方法在锂金属上实现自发还原的石 墨烯涂层。在与 $\mathrm{LiFePO}_{4}$ 组装的软包电池中展现出 良好的循环稳定性和倍率性能(图8b, c)。不仅如 此, 此方法具有一定的可扩展性, 当与卷对卷工艺 结合时, 可以实现连续制造。Cui等 ${ }^{49}$ 通过化学气 相沉积法(CVD)直接在铜箔上生长石墨烯和六方 氮化嗍(h-BN)。首先, 石墨烯和h-BN具有较高的化 学稳定性。其次, 由于层内键合能力强, 石墨烯和 $\mathrm{h}-\mathrm{BN}$ 的单原子层具有高机械强度足以抑制锂枝晶 的生长。此外, h-BN的缺陷为 $\mathrm{Li}^{+}$的传输创造了良 好的通道。同时 $1-10$ 层的h-BN具有很高的柔韧性, 能够容纳金属锂沉积的体积变化。在碳酸盐电解 质中, 当电流密度为 $2 \mathrm{~mA} \cdot \mathrm{cm}^{-2}$ 和沉积量为 5 $\mathrm{mAh} \cdot \mathrm{cm}^{-2}$ 下循环 50 个周期后库伦效率达到 $97 \%$ 。 碳材料具有优异的机械性能, 但是碳质保护膜的 制备过程较为繁琐, 且其与锂金属的界面接触问 题还需进一步探讨。

\section{3 有机-无机复合保护膜}

由于材料自身的特性, 各类保护膜都存在一 定的局限性, 近几年复合保护膜因其能够综合各 方面的优势而备受关注。例如, 聚合物保护膜能够 缓解循环过程中的体积变化, 促进 $\mathrm{Li}^{+}$传输, 但是 由于其机械模量较低, 在长时间的电池循环中抑 制枝晶的效果不尽人意。而机械强度高的无机保 护膜能够弥补聚合物膜的不足。因此, 研究人员一 直致力于开发具有不同保护膜优点的混合保护膜 a

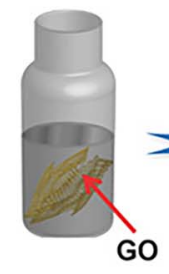

G

Alkali Metal

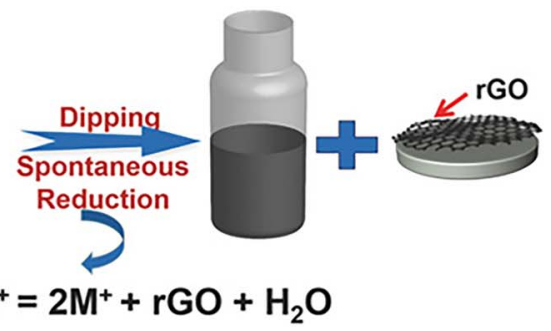

b
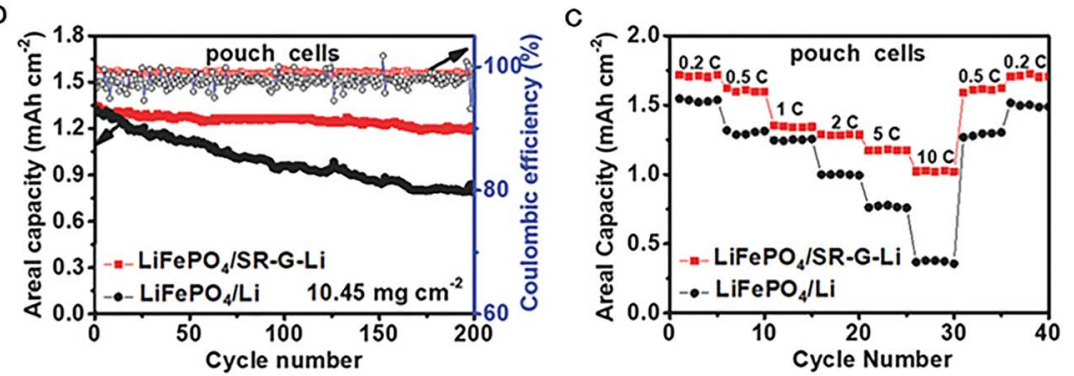

图 8 (a) SR-G-Li 负极的制备示意图; $\mathrm{LiFePO}_{4} / \mathrm{SR}-\mathrm{G}-\mathrm{Li}$ 和 $\mathrm{LiFePO}_{4} / \mathrm{Li}$ 软包电池的电化学性能图:

(b)恒流循环 200 个周期; (c)倍率性能图 88

Fig. 8 (a) A schematic illustration of the fabrication of the SR-G-Li anode; the Electrochemical performance of the pouch cells of $\mathrm{LiFePO}_{4} / \mathrm{SR}-\mathrm{G}-\mathrm{Li}$ and $\mathrm{LiFePO}_{4} / \mathrm{Li}$ of (b) the long-term galvanostatic cycling up to 200 cycles and

(c) rate capability at various current densities ${ }^{88}$. 
用于锂金属负极。

为了综合聚合物保护膜和无机保护膜的优 势, 聚合物-无机复合保护膜应运而生。这类复合 膜通常由无机陶瓷颗粒和聚合物组成。聚偏二氟 乙烯-六氟丙烯(PVDF-HFP) 常用于粘结剂, 是一种 半结晶聚合物, 非晶化的HFP片段有利于提高锂 离子传导率，而剩余的结晶区域则保证了基本的 机械强度。Huang等 ${ }^{89}$ 在PVDF-HFP嵌入 LiF颗粒制 备复合膜 $(\mathrm{APL})$ 作为人造 SEI膜。LiF颗粒的均匀嵌 入使PVDF-HFP膜的杨氏模量从 $0.8 \mathrm{GPa}$ 提高到 $6.72 \mathrm{GPa}$ 。同时 APL具有丰富的中孔 $(2.5-12 \mathrm{~nm})$, 为 $\mathrm{Li}^{+}$的传输提供良好的通道。除此之外, APL还 具有良好的延展性、弹性和热稳定性。与PVDFHFP膜相比, APL膜包覆的电极电化学性能提升、 沉积形貌更加平整均匀(图9)。Qian等90制备了介孔 二氧化硅加固的聚偏二氟乙烯复合膜 (m$\mathrm{SiO}_{2} / \mathrm{PVDF}$ ) 作为锂负极的人工保护膜。由于纯 PVDF膜的离子传导率有限, 引入有序介孔结构的 $\mathrm{SiO}_{2}$ 为电解质渗透和 $\mathrm{Li}^{+}$扩散提供了足够的通道, 而 PVDF则保持机械完整性和化学稳定性。除了在聚 合物膜中嵌入无机陶瓷颗粒, 研究者们还构建了 包含无机层和有机层的双层保护膜, 两者分工合 作, 协同取得优异的电化学性能。Yan等 ${ }^{91}$ 将金属 锂直接浸没于聚磷酸酯 (PPE) 和四氢呋喃(THF) 中，靠近锂金属表面的PPE分子会自发与锂反应， 在锂金属表面形成 $\mathrm{Li}_{3} \mathrm{PO}_{4}$ 层。该无机层抑制了 $\mathrm{Li}$ 与

(a)

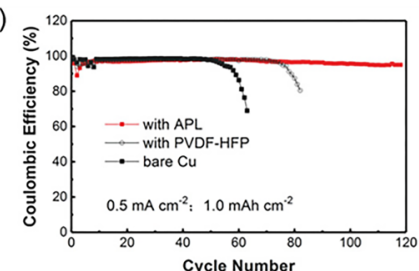

(c)
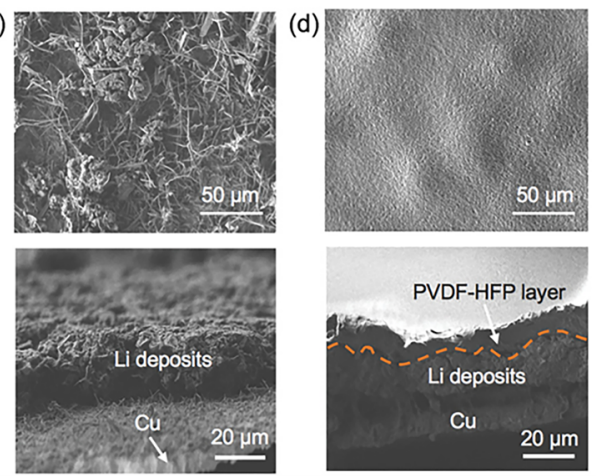

PPE进一步反应, 使其他PPE分子扩散并吸附到无 机层上, 随着THF的蒸发最终形成有机层, 构成典 型的双层结构。通过DFT计算, 表明 $\mathrm{Li}^{+}$与该双层 膜的结合能远低于与酯基SEI膜的结合能(图 10a), 具有更高的锂离子传导率和较小的沉积过电位 (图10b)。通过原子力显微镜测量(AFM), 其杨氏模 量达12 GPa (图10c-e)。在有机保护膜和无机保护 膜的协同作用下, $\mathrm{Li}|| \mathrm{Li}$ 对称电池在电流密度为 2 $\mathrm{mA} \cdot \mathrm{cm}^{-2}$ 的情况下可以稳定循环 800 个周期。 $\mathrm{Wu}$ 等 ${ }^{92}$ 构建了合金/聚合物双层保护膜作为人造 SEI 膜。合金层以三氟化锑 $\left(\mathrm{SbF}_{3}\right)$ 为前驱体, 与金属锂 发生如下反应: $\mathrm{SbF}_{3}+3 \mathrm{Li} \rightarrow \mathrm{Sb}+3 \mathrm{LiF} ; \mathrm{Sb}+$ $3 \mathrm{Li} \rightarrow \mathrm{Li}_{3} \mathrm{Sb}$, 制备了富含 $\mathrm{LiF}$ 的Li-Sb合金层, 该 合金层具有亲锂性和高锂离子传导率。有机层采 用具有高弹性的商用橡胶层, 以适应电池循环时 产生的应力变化。

\section{4 合金保护膜}

通过在金属锂表面覆盖一层金属 $(\mathrm{Si}$ 、In、 $\mathrm{Al}$ 、 $\mathrm{Mg} 、 \mathrm{Sn}$ 等)来调控锂沉积行为 ${ }^{93,94}$, 由于锂的合金 化电位高于电镀电位, 金属层会与锂优先发生合 金化反应，而不是直接镀锂 ${ }^{95}$ 。理想的合金保护层 在循环过程中应保持完整性, 实现可逆的合金/去 合金过程, 同时保证 $\mathrm{Li}^{+}$的快速迁移, 避免枝晶生 长。

Ding 等 ${ }^{96}$ 在金属锂表面扩散录滴，原位形成 $\mathrm{Li}-\mathrm{Hg}$ 保护层实现无枝晶锂负极。该合金层显示出
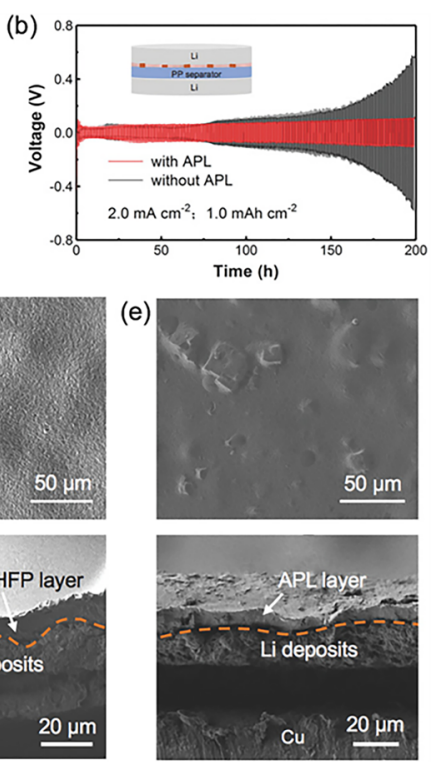

图 9 (a) $\mathrm{Li} \mid \mathrm{Cu}$ 半电池的库伦效率; (b) $\mathrm{Li} \mid \mathrm{Li}$ 对称电池的电压-时间曲线; $0.5 \mathrm{~mA} \cdot \mathrm{cm}^{-2} / 1 \mathrm{mAh} \cdot \mathrm{cm}^{-2}$ 条件下锂沉积 后的扫描电镜俯视图和截面图(c)未保护的铜电极; (d) PVDF-HFP 修饰的铜电极和(e) APL 修饰的铜电极 ${ }^{89}$

Fig. 9 (a) $\mathrm{CE}$ of $\mathrm{Li} \mid \mathrm{Cu}$ half cells; (b) voltage-time curves of symmetric $\mathrm{Li} \mid \mathrm{Li}$ cells; top-view and cross-sectional SEM images of deposited Li (0.5 mA $\left.\cdot \mathrm{cm}^{-2} / 1 \mathrm{mAh} \cdot \mathrm{cm}^{-2}\right)$ on (c) bare Cu, (d) PVDF-HFP-modified Cu, and (e) APL-modified Cu foils at a current density of $0.5 \mathrm{~mA} \cdot \mathrm{cm}^{-2} 89$. 


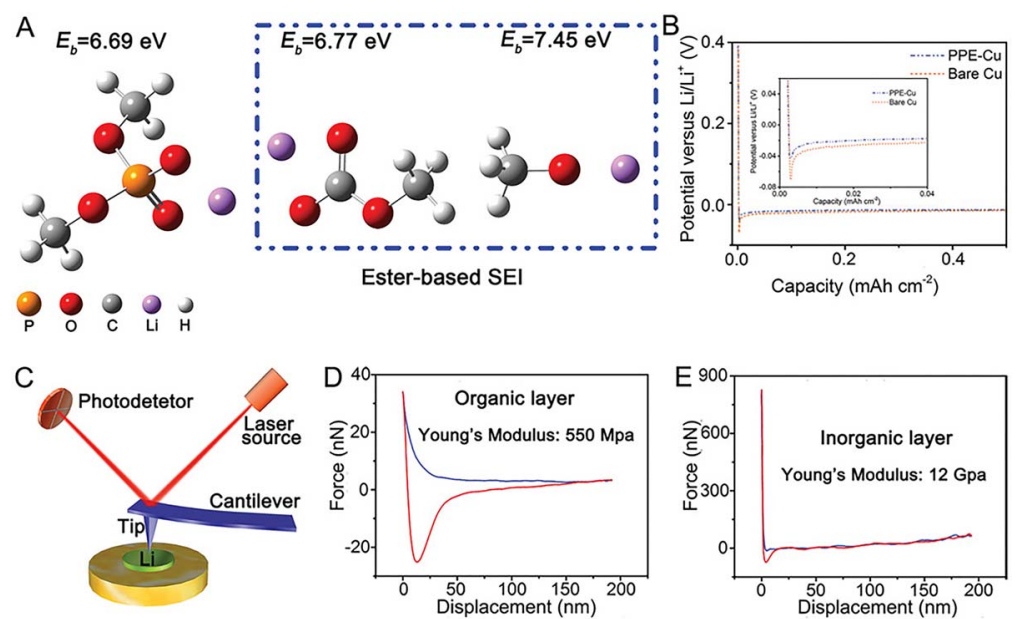

图 10 (a) $\mathrm{Li}^{+}$与双层膜(左)和酯基 SEI 膜(右)之间的原子结构和结合能; (b) $\mathrm{Li}$ 在 $\mathrm{Cu}$ 电极和 PPE-Cu 电极的 沉积过电位; (c) 原子力显微镜(AFM)工作原理示意图; 力-距离曲线和对应的杨氏模量(d)有机层和(e)无机层 ${ }^{91}$ Fig. 10 (a) Atomic structure and binding energy between Li cation and the dual-layered film (left), ester-based SEI (right); (b) the overpotential of Li plating on Cu and PPE-Cu substrates; (c) a schematic diagram for the operational principle of AFM; the force-distance curves and the corresponding Young's modulus values of the (d) organic layer and (e) inorganic layer in the dual-layered film ${ }^{91}$.

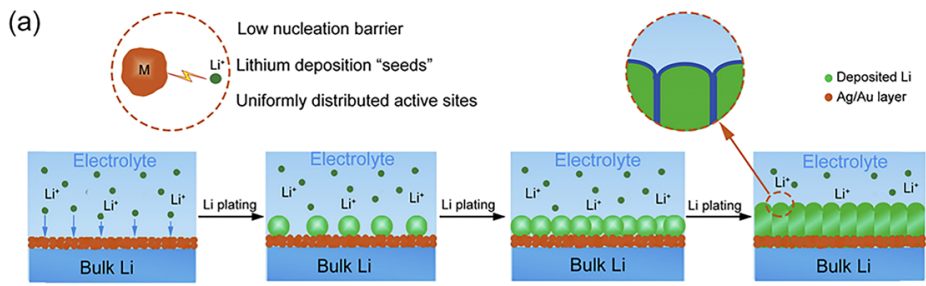

(b)
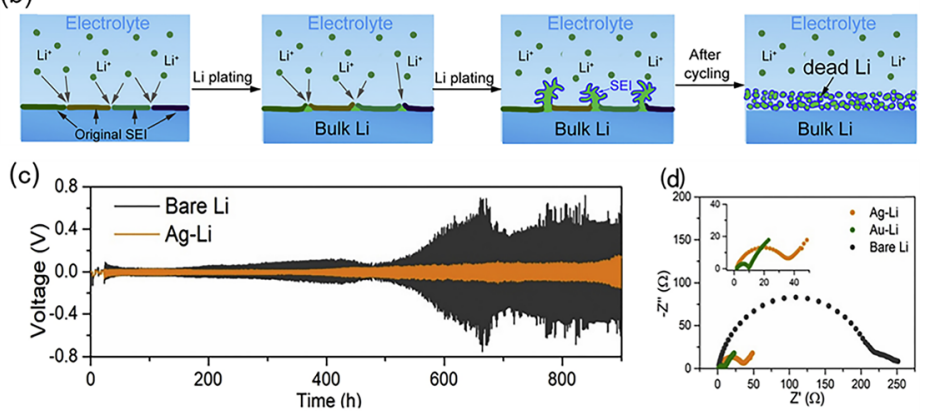

图 11 锂沉积过程示意图(a) $\mathrm{Ag}(\mathrm{Au})-\mathrm{Li}$ 负极和(b)未保护的锂负极; (c)在 $1 \mathrm{~mA} \cdot \mathrm{cm}^{-2} / 1 \mathrm{mAh} \cdot \mathrm{cm}^{-2}$ 下 $\mathrm{Ag}-\mathrm{Li}$ 电极和 未保护的锂电极的时间-电压分布图; (d) Ag-Li、Au-Li 和 $\mathrm{Li}$ 对称电池的电化学阻抗谱图 99

Fig. 11 Schematic illustration of the Li deposition process on the (a) $\mathrm{Ag}(\mathrm{Au})$-Li anode and (b) pristine Li anode;

(c) time-voltage profiles of $\mathrm{Li}$ plating/stripping in $\mathrm{Ag}$ - $\mathrm{Li}$ and bare $\mathrm{Li}$ symmetric cells at $1 \mathrm{~mA} \cdot \mathrm{cm}^{-2}$ with a $\mathrm{Li}$ deposition capacity of $1 \mathrm{mAh} \cdot \mathrm{cm}^{-2}$; (d) electrochemical impedance spectra (EIS) of Ag-Li, Au-Li and Li symmetric cells ${ }^{99}$.

优异的合金化能力以及高柔韧性，可以有效缓解 锂负极循环过程中的应力, 无论是对称电池还是 和 $\mathrm{LiFePO}_{4}$ 组成的全电池中都展现出优异的循环 寿命。 $\mathrm{Au}^{97}$ 和 $\mathrm{Ag}^{98}$ 具有良好的亲锂性, Qian等 ${ }^{99}$ 通 过金属锂与金属离子溶液 $\left(\mathrm{Au}^{3+}\right.$ 或 $\left.\mathrm{Ag}^{2+}\right)$ 之间的原 位置换反应在锂䈃表面形成亲锂的金属层。 $\mathrm{Au} / \mathrm{Ag}$ 在锂沉积前发生合金化反应形成合金层，提供丰 富的成核位点, 降低锂成核势垒, 在沉积过程中诱
导锂均匀成核, 并优先以球状和柱状的形态沉积 (图11a，b)。其对称电池在电流密度为 $1 \mathrm{~mA} \cdot \mathrm{cm}^{-2}$ 、 沉积量为 $1 \mathrm{mAh} \cdot \mathrm{cm}^{-2}$ 的条件下稳定循环超过 900 $\mathrm{h}$, 且阻抗明显小于未保护的锂负极 (图 $11 \mathrm{c}, \mathrm{d}$ )。 $\mathrm{He}$ 等 ${ }^{100}$ 将平均厚度约为 $160 \mathrm{~nm}$ 的超薄铝䇴裁剪成圆 片, 直接通过物理按压覆在锂金属表面, 在电池充 放电过程中进行电化学合金化形成Li-Al合金保护 膜, 实现锂负极无锂枝晶形态。合金类保护膜通过 
降低成核过电位有效调控锂沉积行为, 当与聚合 物材料进行复合时能够提高其力学性能。但是, 目 前合金保护膜在锂金属电池中的作用机制尚不明 确, 还需深入研究。

\section{4 总结与展望}

为实现高能量密度电池, 学术界和工业界对 锂金属负极的研究越来广泛, 但是由于枝晶生长 带来的巨大挑战阻碍了锂金属负极实现商业化。在 电池循环过程中, 低模量的SEI膜直接影响了锂沉 积过程, 因此构建稳定的SEI膜至关重要。通过在 锂负极表面构建人造保护膜是提高锂离子传导率 和解决枝晶问题的一种有效策略, 其中高柔韧性 的聚合物保护膜能够缓解电池循环的体积效应; 高 机械强度的无机保护膜能够有效抑制枝晶生长; 有 机-无机复合保护膜综合两者优势, 实现优异的电 化学性能; 合金保护膜与金属锂发生合金化反应, 降低锂成核势垒, 诱导均匀的锂沉积。尽管如此, 构建人造保护膜仍然存在以下挑战:

(1)由于目前人们对SEI膜的物理结构和化学 成分还没有足够的认识, 在构建人造膜时其厚度 和离子传导率等方面的设计依旧存在一定的缺 陷。

(2)大部分人造保护膜在组装扣式电池时使用 的锂片是远远过量的, 且在进行电化学性能测试 时采用较小的电流密度和较小的面容量。此条件 下的电化学性能与商业实际测试条件下的性能偏 差较大, 无法检验所提出保护膜策略的可行性。

(3)开发新的制备方法或采用先进的沉积技 术, 严格控制保护膜的厚度精度和均匀性。同时结 合原位表征方法, 包括扫描电子显微镜、透射电子 显微镜、原子力显微镜以及其他先进的表征方法, 深入了解保护膜与锂负极的界面性质, 继续探索 两者之间的相互作用机理, 以更好的理解 $\mathrm{Li}^{+}$的沉 积变化过程。

人造保护膜应用于锂金属负极的研究获得广 泛关注, 其对解决枝晶问题具有重要意义, 但是目 前各类保护膜依旧存在一定的局限性, 今后可以 结合物理生物等领域, 构建出理想的保护膜, 早日 实现高比能锂金属电池的应用。

\section{References}

(1) Evarts, E. C. Nature 2015, 526, S93. doi: 10.1038/526S93a

(2) Janek, J.; Zeier, W. G. Nat. Energy 2016, 1, 16141. doi: 10.1038/nenergy.2016.141

(3) Li, P.; Hwang, J. Y.; Sun, Y. K. ACS Nano 2019, 13, 2624. doi: $10.1021 /$ acsnano.9b00169

(4) Cheng, X. B.; Zhang, R.; Zhao, C. Z.; Zhang, Q. Chem. Rev. 2017, 117, 10403. doi: 10.1021/acs.chemrev.7b00115

(5) Sun, Y.; Ma, P.; Liu, L.; Chen, J.; Zhang, X.; Lang, J.; Yan, X. Sol. RRL 2018, 2, 1800223. doi: 10.1002/solr.201800223

(6) Sun, Y.; Yan, X. Sol. RRL 2017, 1, 1700002. doi: 10.1002/solr.201700002

(7) Tarascon, J. M.; Armand, M. Nature 2001, 414, 359. doi: $10.1038 / 35104644$

(8) Xu, W.; Wang, J.; Ding, F.; Chen, X.; Nasybulin, E.; Zhang, Y.; Zhang, J. G. Energy Environ. Sci. 2014, 7, 513. doi: 10.1039/C3EE40795K

(9) Niu, C.; Pan, H.; Xu, W.; Xiao, J.; Zhang, J. G.; Luo, L.; Wang, C.; Mei, D.; Meng, J.; Wang, X.; et al. Nat. Nanotechnol. 2019, 14, 594. doi: 10.1038/s41565-019-0427-9

(10) Bruce, P. G.; Freunberger, S. A.; Hardwick, L. J.; Tarascon, J. M. Nat. Mater. 2012, 11, 19. doi: 10.1038/nmat3191

(11) Bruce, P. G.; Hardwick, L. J.; Abraham, K. M. MRS Bull. 2011, 36, 506. doi: $10.1557 / \mathrm{mrs} .2011 .157$

(12) Xu, X.; Wang, S.; Wang, H.; Hu, C.; Jin, Y.; Liu, J.; Yan, H. J. Energy Chem. 2018, 27, 513. doi: 10.1016/j.jechem.2017.11.010

(13) Zhang, X. Q.; Zhao, C. Z.; Huang, J. Q.; Zhang, Q. Engineering 2018, 4, 831. doi: 10.1016/j.eng.2018.10.008

(14) Lin, D.; Liu, Y.; Cui, Y. Nat. Nanotechnol. 2017, 12, 194. doi: $10.1038 /$ nnano.2017.16

(15) Feng, Y.; Zhang, C.; Li, B.; Xiong, S.; Song, J. J. Mater. Chem. A 2019, 7, 6090. doi: 10.1039/C8TA10779C

(16) Qie, L.; Zu, C.; Manthiram, A. Adv. Energy Mater. 2016, 6, 1502459. doi: 10.1002/aenm.201502459

(17) Zhang, Y.; Luo, W.; Wang, C.; Li, Y.; Chen, C.; Song, J.; Dai, J.; Hitz, E. M.; Xu, S.; Yang, C.; et al. Proc. Natl. Acad. Sci. 2017, 114, 3584. doi: 10.1073/pnas.1618871114

(18) Chazalviel, J. N. Phys. Rev. A 1990, 42, 7355. doi: 10.1103/PhysRevA.42.7355

(19) Brissot, C.; Rosso, M.; Chazalviel, J. N.; Lascaud, S. J. Power Sources 1999, 81-82, 925. doi: 10.1016/S0378-7753(98)00242-0

(20) Ding, F.; Xu, W.; Graff, G. L.; Zhang, J.; Sushko, M. L.; Chen, X.; Shao, Y.; Engelhard, M. H.; Nie, Z.; Xiao, J.; et al. J. Am. Chem. Soc. 2013, 135, 4450. doi: 10.1021/ja312241y

(21) Enze, L. J. Phys. D: Appl. Phys. 1986, 19, 1. doi: 10.1088/0022-3727/19/1/005

(22) Liu, F. F.; Zhang, Z. W.; Ye, S. F.; Yao, Y.; Yu, Y. Acta Phys. -Chim. Sin. 2021, 37, 2006021. [刘凡凡, 张志文, 叶淑芬, 姚雨, 余彦. 物理化学学报, 2021, 37, 2006021.] doi: 10.3866/PKU.WHXB202006021

(23) Kushima, A.; So, K. P.; Su, C.; Bai, P.; Kuriyama, N.; Maebashi, T.; 
Fujiwara, Y.; Bazant, M. Z.; Li, J. Nano Energy 2017, 32, 271.

doi: 10.1016/j.nanoen.2016.12.001

(24) Jäckle, M.; Groß, A. J. Chem. Phys. 2014, 141, 174710. doi: $10.1063 / 1.4901055$

(25) Wang, D.; Zhang, W.; Zheng, W.; Cui, X.; Rojo, T.; Zhang, Q. Adv. Sci. 2017, 4, 1600168. doi: 10.1002/advs.201600168

(26) Yamaki, J.; Tobishima, S.; Hayashi, K.; Saito, K.; Nemoto, Y.; Arakawa, M. J. Power Sources 1998, 74, 219.

doi: 10.1016/S0378-7753(98)00067-6

(27) Wang, G.; Xiong, X.; Xie, D.; Fu, X.; Ma, X.; Li, Y.; Liu, Y.; Lin, Z.; Yang, C.; Liu, M. Energy Storage Mater. 2019, 23, 701. doi: 10.1016/j.ensm.2019.02.026

(28) Fu, X.; Wang, G.; Dang, D.; Liu, Q.; Xiong, X.; Wu, C. J. Mater. Chem. A 2019, 7, 25003. doi: 10.1039/C9TA09068A

(29) Ran, Q.; Sun, T. Y.; Han, C. Y.; Zhang, H. N.; Yan, J.; Wang, J. L. Acta Phys. -Chim. Sin. 2020, 36, 1912068. [由琴, 孙天霷, 韩冲宇, 张浩楠, 颜剑, 汪靖伦. 物理化学学报, 2020, 36, 1912068.] doi: 10.3866/PKU.WHXB201912068

(30) Luo, C.; Ji, X.; Chen, J.; Gaskell, K. J.; He, X.; Liang, Y.; Jiang, J.; Wang, C. Angew. Chem. 2018, 130, 8703. doi: 10.1002/ange. 201804068

(31) Zhang, B.; Tan, R.; Yang, L.; Zheng, J.; Zhang, K.; Mo, S.; Lin, Z.; Pan, F. Energy Storage Mater. 2018, 10, 139. doi: 10.1016/j.ensm.2017.08.015

(32) Luo, W.; Gong, Y.; Zhu, Y.; Fu, K. K.; Dai, J.; Lacey, S. D.; Wang, C.; Liu, B.; Han, X.; Mo, Y.; et al. J. Am. Chem. Soc. 2016, 138, 12258. doi: $10.1021 /$ jacs.6b06777

(33) Gu, J.; Zhu, Q.; Shi, Y.; Chen, H.; Zhang, D.; Du, Z.; Yang, S. ACS Nano 2020, 14, 891. doi: 10.1021/acsnano.9b08141

(34) Xu, B.; Zhai, H.; Liao, X.; Qie, B.; Mandal, J.; Gong, T.; Tan, L.; Yang, X.; Sun, K.; Cheng, Q.; et al. Energy Storage Mater. 2019, 17, 31. doi: 10.1016/j.ensm.2018.11.035

(35) Kim, M. S.; Ryu, J. H.; Deepika; Lim, Y. R.; Nah, I. W.; Lee, K. R.; Archer, L. A.; Il Cho, W. Nat. Energy 2018, 3, 889. doi: 10.1038/s41560-018-0237-6

(36) Liu, Y.; Lin, D.; Yuen, P. Y.; Liu, K.; Xie, J.; Dauskardt, R. H.; Cui, Y. Adv. Mater. 2017, 29, 1605531. doi: 10.1002/adma.201605531

(37) Deng, K.; Han, D.; Ren, S.; Wang, S.; Xiao, M.; Meng, Y. J. Mater. Chem. A 2019, 7, 13113. doi: 10.1039/C9TA02407G

(38) Zhang, C.; Lyu, R.; Lv, W.; Li, H.; Jiang, W.; Li, J.; Gu, S.; Zhou, G.; Huang, Z.; Zhang, Y.; et al. Adv. Mater. 2019, 31, 1904991. doi: 10.1002/adma.201904991

(39) Chi, S.; Liu, Y.; Song, W.; Fan, L.; Zhang, Q. Adv. Funct. Mater. 2017, 27, 1700348. doi: 10.1002/adfm.201700348

(40) Luo, L.; Li, J.; Yaghoobnejad Asl, H.; Manthiram, A. Adv. Mater. 2019, 31, 1904537. doi: 10.1002/adma.201904537
(41) Wang, M. Q.; Peng, Z.; Lin, H.; Li, Z. D.; Liu, J.; Ren, Z. M.; He, H. Y.; Wang, D. Y. Acta Phys. -Chim. Sin. 2021, 37, 2007016. [王木钦, 彭哲, 林欢, 李振东, 刘健, 任重民, 何海勇, 王德宇. 物理化学 学报, 2021, 37, 2007016.] doi: 10.3866/PKU.WHXB202007016

(42) Chen, X.; Hou, T. Z.; Li, B.; Yan, C.; Zhu, L.; Guan, C.; Cheng, X. B.; Peng, H. J.; Huang, J. Q.; Zhang, Q. Energy Storage Mater. 2017, 8, 194. doi: 10.1016/j.ensm.2017.01.003

(43) Tao, R.; Bi, X.; Li, S.; Yao, Y.; Wu, F.; Wang, Q.; Zhang, C.; Lu, J. ACS Appl. Mater. Interfaces 2017, 9, 7003. doi: 10.1021/acsami.6b13859

(44) Huang, G.; Han, J.; Zhang, F.; Wang, Z.; Kashani, H.; Watanabe, K.; Chen, M. Adv. Mater. 2019, 31, 1805334. doi: 10.1002/adma.201805334

(45) Liu, S.; Zhang, X.; Li, R.; Gao, L.; Luo, J. Energy Storage Mater. 2018, 14, 143. doi: 10.1016/j.ensm.2018.03.004

(46) Cheng, X.; Zhang, R.; Zhao, C.; Wei, F.; Zhang, J. G.; Zhang, Q. Adv. Sci. 2016, 3, 1500213. doi: 10.1002/advs.201500213

(47) Rui, K.; Wen, Z.; Lu, Y.; Jin, J.; Shen, C. Adv. Energy Mater. 2015, 5, 1401716. doi: 10.1002/aenm.201401716

(48) Zheng, J.; Engelhard, M. H.; Mei, D.; Jiao, S.; Polzin, B. J.; Zhang, J. G.; Xu, W. Nat. Energy 2017, 2, 17012. doi: 10.1038/nenergy.2017.12

(49) Yan, K.; Lee, H. W.; Gao, T.; Zheng, G.; Yao, H.; Wang, H.; Lu, Z.; Zhou, Y.; Liang, Z.; Liu, Z.; et al. Nano Lett. 2014, 14, 6016. doi: $10.1021 / \mathrm{n} 1503125 \mathrm{u}$

(50) Chen, L.; Connell, J. G.; Nie, A.; Huang, Z.; Zavadil, K. R.; Klavetter, K. C.; Yuan, Y.; Sharifi-Asl, S.; Shahbazian-Yassar, R.; Libera, J. A.; et al. J. Mater. Chem. A 2017, 5, 12297. doi: 10.1039/C7TA03116E

(51) Kushima, A.; So, K. P.; Su, C.; Bai, P.; Kuriyama, N.; Maebashi, T.; Fujiwara, Y.; Bazant, M. Z.; Li, J. Nano Energy 2017, 32, 271. doi: 10.1016/j.nanoen.2016.12.001

(52) Zheng, G.; Wang, C.; Pei, A.; Lopez, J.; Shi, F.; Chen, Z.; Sendek, A. D.; Lee, H. W.; Lu, Z.; Schneider, H.; et al. ACS Energy Lett. 2016, 1, 1247. doi: 10.1021/acsenergylett.6b00456

(53) Choi, S. M.; Kang, I. S.; Sun, Y. K.; Song, J. H.; Chung, S. M.; Kim, D. W. J. Power Sources 2013, 244, 363. doi: 10.1016/j.jpowsour.2012.12.106

(54) Zhu, J.; Li, P.; Chen, X.; Legut, D.; Fan, Y.; Zhang, R.; Lu, Y.; Cheng, X.; Zhang, Q. Energy Storage Mater. 2019, 16, 426. doi: 10.1016/j.ensm.2018.06.023

(55) Bai, M.; Xie, K.; Yuan, K.; Zhang, K.; Li, N.; Shen, C.; Lai, Y.; Vajtai, R.; Ajayan, P.; Wei, B. Adv. Mater. 2018, 30, 1801213. doi: 10.1002/adma.201801213

(56) Wang, G.; Xiong, X.; Xie, D.; Fu, X.; Lin, Z.; Yang, C.; Zhang, K.; Liu, M. ACS Appl. Mater. Interfaces 2019, 11, 4962. 
doi: $10.1021 /$ acsami.8b18101

(57) Zhou, D.; Liu, R.; He, Y.; Li, F.; Liu, M.; Li, B.; Yang, Q.; Cai, Q.; Kang, F. Adv. Energy Mater. 2016, 6, 1502214. doi: 10.1002/aenm.201502214

(58) Jang, E. K.; Ahn, J.; Yoon, S.; Cho, K. Y. Adv. Funct. Mater. 2019, 29, 1905078. doi: 10.1002/adfm.201905078

(59) Zhang, Z.; Zhang, L.; Liu, Y.; Yang, T.; Wang, Z.; Yan, X.; Yu, C. J. Mater. Chem. A 2019, 7, 23173. doi: 10.1039/C9TA08415K

(60) Liang, X.; Pang, Q.; Kochetkov, I. R.; Sempere, M. S.; Huang, H.; Sun, X.; Nazar, L. F. Nat. Energy 2017, 2, 17119. doi: 10.1038/nenergy.2017.119

(61) Wang, G.; Xiong, X.; Lin, Z.; Zheng, J.; Fenghua, Z.; Li, Y.; Liu, Y.; Yang, C.; Tang, Y.; Liu, M. Nanoscale 2018, 10, 10018. doi: $10.1039 / \mathrm{C} 8 \mathrm{NR} 01995 \mathrm{~A}$

(62) Wu, H.; Yao, Z.; Wu, Q.; Fan, S.; Yin, C.; Li, C. J. Mater. Chem. A 2019, 7, 22257. doi: 10.1039/C9TA09146G

(63) Sun, Y.; Zhao, Y.; Wang, J.; Liang, J.; Wang, C.; Sun, Q.; Lin, X.; Adair, K. R.; Luo, J.; Wang, D.; et al. Adv. Mater. 2019, 31, 1806541. doi: 10.1002/adma.201806541

(64) Wu, H.; Wu, Q.; Chu, F.; Hu, J.; Cui, Y.; Yin, C.; Li, C. J. Power Sources 2019, 419, 72. doi: 10.1016/j.jpowsour.2019.02.033

(65) Wang, G.; Chen, C.; Chen, Y.; Kang, X.; Yang, C.; Wang, F.; Liu, Y.; Xiong, X. Angew. Chem. Int. Ed. 2020, 59, 2055. doi: 10.1002/anie.201913351

(66) Lopez, J.; Pei, A.; Oh, J. Y.; Wang, G. J. N.; Cui, Y.; Bao, Z. J. Am. Chem. Soc. 2018, 140, 11735. doi: 10.1021/jacs.8b06047

(67) Monroe, C.; Newman, J. J. Electrochem. Soc. 2003, 150, A1377. doi: $10.1149 / 1.1606686$

(68) Stone, G. M.; Mullin, S. A.; Teran, A. A.; Hallinan, D. T.; Minor, A. M.; Hexemer, A.; Balsara, N. P. J. Electrochem. Soc. 2012, 159, A222. doi: 10.1149/2.030203jes

(69) Pei, A.; Zheng, G.; Shi, F.; Li, Y.; Cui, Y. Nano Lett. 2017, 17, 1132. doi: 10.1021/acs.nanolett.6b04755

(70) Huang, S.; Zhang, W.; Ming, H.; Cao, G.; Fan, L. Z.; Zhang, H. Nano Lett. 2019, 19, 1832. doi: 10.1021/acs.nanolett.8b04919

(71) Cui, Y. Acta Phys. -Chim. Sin. 2019, 35, 661. [崔屹. 物理化学学报, 2019, 35, 661.] doi:10.3866/PKU.WHXB201809053

(72) Xiang, H.; Chen, J.; Li, Z.; Wang, H. J. Power Sources 2011, 196, 8651. doi: 10.1016/j.jpowsour.2011.06.055

(73) He, M.; Zhang, X.; Jiang, K.; Wang, J.; Wang, Y. ACS Appl. Mater. Interfaces 2015, 7, 738. doi: 10.1021/am507145h

(74) Wang, G.; Xiong, X.; Zou, P.; Fu, X.; Lin, Z.; Li, Y.; Liu, Y.; Yang, C.; Liu, M. Chem. Eng. J. 2019, 378, 122243. doi: 10.1016/j.cej.2019.122243

(75) Kozen, A. C.; Lin, C. F.; Pearse, A. J.; Schroeder, M. A.; Han, X.; Hu, L.; Lee, S. B.; Rubloff, G. W.; Noked, M. ACS Nano 2015, 9 ,
5884 doi: $10.1021 /$ acsnano.5b02166

(76) Kazyak, E.; Wood, K. N.; Dasgupta, N. P. Chem. Mater. 2015, 27, 6457. doi: 10.1021/acs.chemmater.5b02789

(77) Umeda, G. A.; Menke, E.; Richard, M.; Stamm, K. L.; Wudl, F.; Dunn, B. J. Mater. Chem. 2011, 21, 1593. doi: 10.1039/C0JM02305A

(78) Ma, G.; Wen, Z.; Wu, M.; Shen, C.; Wang, Q.; Jin, J.; Wu, X. Chem. Commun. 2014, 50, 14209. doi: 10.1039/C4CC05535G

(79) Zhao, J.; Liao, L.; Shi, F.; Lei, T.; Chen, G.; Pei, A.; Sun, J.; Yan, K.; Zhou, G.; Xie, J.; et al. J. Am. Chem. Soc. 2017, 139, 11550. doi: $10.1021 /$ jacs. $7 \mathrm{~b} 05251$

(80) Lin, Y.; Wen, Z.; Liu, J.; Wu, D.; Zhang, P.; Zhao, J. J. Energy Chem. 2021, 55, 129. doi: 10.1016/j.jechem.2020.07.003

(81) Li, N.; Yin, Y.; Yang, C.; Guo, Y. Adv. Mater. 2016, 28, 1853. doi: 10.1002/adma.201504526

(82) Lin, L.; Liang, F.; Zhang, K.; Mao, H.; Yang, J.; Qian, Y. J. Mater. Chem. A 2018, 6, 15859. doi: 10.1039/C8TA05102J

(83) Liu, F.; Wang, L.; Zhang, Z.; Shi, P.; Feng, Y.; Yao, Y.; Ye, S.; Wang, H.; Wu, X.; Yu, Y. Adv. Funct. Mater. 2020, 30, 2001607. doi: 10.1002/adfm.202001607

(84) Herbert, E. G.; Tenhaeff, W. E.; Dudney, N. J.; Pharr, G. M. Thin Solid Films 2011, 520, 413. doi: 10.1016/j.tsf.2011.07.068

(85) Wang, W.; Yue, X.; Meng, J.; Wang, J.; Wang, X.; Chen, H.; Shi, D.; Fu, J.; Zhou, Y.; Chen, J.; et al. Energy Storage Mater. 2019, 18, 414. doi: 10.1016/j.ensm.2018.08.010

(86) Zhang, Y. J.; Liu, X. Y.; Bai, W. Q.; Tang, H.; Shi, S. J.; Wang, X. L.; Gu, C. D.; Tu, J. P. J. Power Sources 2014, 266, 43. doi: 10.1016/j.jpowsour.2014.04.147

(87) Zheng, G.; Lee, S. W.; Liang, Z.; Lee, H. W.; Yan, K.; Yao, H.; Wang, H.; Li, W.; Chu, S.; Cui, Y. Nat. Nanotechnol. 2014, 9, 618. doi: 10.1038/nnano.2014.152

(88) Bai, M.; Xie, K.; Yuan, K.; Zhang, K.; Li, N.; Shen, C.; Lai, Y.; Vajtai, R.; Ajayan, P.; Wei, B. Adv. Mater. 2018, 30, 1801213. doi: 10.1002/adma.201801213

(89) Xu, R.; Zhang, X.; Cheng, X.; Peng, H.; Zhao, C.; Yan, C.; Huang, J. Adv. Funct. Mater. 2018, 28, 1705838. doi: 10.1002/adfm.201705838

(90) Wu, C.; Guo, F.; Zhuang, L.; Ai, X.; Zhong, F.; Yang, H.; Qian, J. ACS Energy Lett. 2020, 5, 1644. doi: 10.1021/acsenergylett.0c00804

(91) Liu, X.; Liu, J.; Qian, T.; Chen, H.; Yan, C. Adv. Mater. 2020, 32, 1902724. doi: 10.1002/adma.201902724

(92) Zhang, Y.; Wang, G.; Tang, L.; Wu, J.; Guo, B.; Zhu, M.; Wu, C.; Dou, S. X.; Wu, M. J. Mater. Chem. A 2019, 7, 25369. doi: 10.1039/C9TA09523C

(93) Kim, H.; Lee, J. T.; Lee, D. C.; Oschatz, M.; Cho, W. Il; Kaskel, S.; Yushin, G. Electrochem. Commun. 2013, 36, 38. doi: 10.1016/j.elecom.2013.09.002 
(94) Jiang, Y.; Jiang, J.; Wang, Z.; Han, M.; Liu, X.; Yi, J.; Zhao, B.; Sun, X.; Zhang, J. Nano Energy 2020, 70, 104504.

doi: 10.1016/j.nanoen.2020.104504

(95) Obrovac, M. N.; Chevrier, V. L. Chem. Rev. 2014, 114, 11444. doi: $10.1021 / \mathrm{cr} 500207 \mathrm{~g}$

(96) He, G.; Li, Q.; Shen, Y.; Ding, Y. Angew. Chem. Int. Ed. 2019, 58, 18466. doi: 10.1002/anie. 201911800

(97) Wang, H.; Li, Y.; Li, Y.; Liu, Y.; Lin, D.; Zhu, C.; Chen, G.; Yang, A.; Yan, K.; Chen, H.; et al. Nano Lett. 2019, 19, 1326. doi: 10.1021/acs.nanolett.8b04906

(98) Yang, C.; Yao, Y.; He, S.; Xie, H.; Hitz, E.; Hu, L. Adv. Mater. 2017, 29, 1702714. doi: 10.1002/adma.201702714

(99) Guo, F.; Wu, C.; Chen, H.; Zhong, F.; Ai, X.; Yang, H.; Qian, J. Energy Storage Mater. 2020, 24, 635. doi: 10.1016/j.ensm.2019.06.010

(100) Wu, L.; He, G.; Ding, Y. J. Mater. Chem. A 2019, 7, 25415. doi: 10.1039/C9TA09464D 